Chair for Management Sciences and Energy Economics University of Duisburg-Essen

EWL Working Paper No. 6

\title{
Valuing Fuel Diversification In Optimal InVestment Policies For Electricity Generation Portfolios
}

by

Malte Sunderkötter

and

Christoph Weber

November 2009 


\title{
Valuing fuel diversification in optimal investment policies for electricity generation portfolios
}

\author{
by Malte Sunderkötter and Christoph Weber
}

\begin{abstract}
Optimal capacity allocation for investments in electricity generation assets can be deterministically derived by comparing technology specific long-term and short-term marginal costs. In an uncertain market environment, Mean-Variance Portfolio (MVP) theory provides a consistent framework to valuate financial risks in power generation portfolios that allows to derive the efficient fuel mix of a system portfolio with different generation technologies from a welfare maximization perspective.

Because existing literature on MVP applications in electricity generation markets uses predominantly numerical methods to characterize portfolio risks, this article presents a novel analytical approach combining conceptual elements of peak-load pricing and MVP theory to derive optimal portfolios consisting of an arbitrary number of plant technologies given uncertain fuel prices. For this purpose, we provide a static optimization model which allows to fully capture fuel price risks in a mean variance portfolio framework. The analytically derived optimality conditions contribute to a much better understanding of the optimal investment policy and its risk characteristics compared to existing numerical methods. Furthermore, we demonstrate an application of the proposed framework and results to the German electricity market which has not yet been treated in MVP literature on electricity markets.
\end{abstract}

Keywords : power plant investments, peak load pricing, mean-variance portfolio theory, fuel mix diversification JEL-Classification : G11, L94, Q43, C44

DIPL. WI.-ING. MALTE SUNDERKÖTTER

Chair for Management Sciences and Energy Economics, University of Duisburg-Essen (Campus Essen)

Universitätsstr. 11, 45117 Essen

++49 - (0)69 / 9150-2192

www.ewl.wiwi.uni-due.de sunderkoetter.malte@bcg.com
Prof. DR. CRISTOPH Weber

Chair for Management Sciences and Energy Economics, University of Duisburg-Essen (Campus Essen)

Universitätsstr. 11, 45117 Essen

++49 - (0)2 01 / 183-2966

www.ewl.wiwi.uni-due.de

christoph.weber@uni-due.de

The authors are solely responsible for the contents which do not necessarily represent the opinion of the Chair for Management Sciences and Energy Economics. 


\section{Introduction}

In the next decades, the European power industry will face an immense need for investments to renew and extend its power plant fleet. ${ }^{2}$ In parallel, EU and several national states have implemented different development schemes which politically influence investment decisions and thereby the fuel mix of the respective country. ${ }^{3}$ For an economic assessment of power plant investments and for quantifying the effect of subsidies on the long-term optimal system fuel mix from a welfare perspective, it is not only crucial to valuate expected total life-cycle costs, but also the economic risks conveyed with investment and operation of the plant fleet. With fuel prices fluctuating considerably, cost volatility becomes a severe risk that influences the advantageousness of an investment and therewith the system optimal fuel mix.

Mean-Variance Portfolio (MVP) theory has become a clear, elegant and proven framework to capture the two aspects of risk and return in a single decision support model since the work of Markowitz (1952, 1959) has set the stage for MVP theory in financial markets. Markowitz' theory builds on the premise that a compound portfolio of assets shows reduced variance characteristics in case each pair of assets shows only imperfect correlation. Similarly, portfolio cost risks can be reduced in a portfolio of well-chosen generation technology options as a result of less than perfect correlations between their cost characteristics. This approach can also be applied to derive efficient electricity generation portfolios from a risk-cost perspective.

Standard models use numerical simulation techniques to derive efficient power generation portfolios. This methodology makes it possible to solve even very complex optimization problems with numerous plants and technologies, but it naturally complicates the understanding for the exact interplay of the different input parameters. This article provides an easily interpretable analytical optimality condition for efficient generation portfolios that can contribute to a better understanding of MVP in electricity applications. The proposed model is then applied to the German electricity market.

This paper is structured as follows: We begin by reviewing relevant literature and briefly discussing the selection of adequate risk and return measures in Section 2. Section 3 contains the formulation and proposes a general solution approach of the portfolio optimization problem. For a simplified case with two technologies, optimality conditions are analytically derived and discussed. The insights from the theoretical model are demonstrated and interpreted in a case study on the German generation portfolio in Section 4. Moreover in contrast to standard MVP models, the merit order of the different power plants and the resulting differences in operation hours are taken into account, using the framework of the peak-load pricing model going back to Boiteux (1960). The article concludes in Section 5 with a summary of key results and an outlook of related interesting areas for future research.

\footnotetext{
${ }^{2}$ The IEA (2008) estimates gross generation capacity additions in European OECD countries of 221GW between 2007 and 2015 and another 465GW between 2016 and 2030 .

${ }^{3} \mathrm{An}$ example for such a subsidy is the the Renewable Energy Law enacted by the German parliament in 2000.
} 


\section{Portfolio diversification}

\subsection{Literature review}

Existing work on portfolio and investment risk management in electricity generation markets can be grouped into two main categories: One stream of research quantitatively approaches the problem by adopting Mean-Variance Portfolio (MVP) theory from finance, whereas the other is based on the concept of diversity which was first explored in natural science applications.

Based on the work of Markowitz (1952, 1959), Bar-Lev and Katz (1976) adopted the MVP approach to long-term portfolio optimization in electricity markets. Their article focuses on the fuel-cost optimization of fossil plants in the U.S. electric utility industry. The authors introduce a "cost-risk" framework instead of the return-risk applied in MVP.

With the emergence of simulation techniques in economic research and the progressing liberalization of electricity markets in different countries, the topic reappeared on the academic agenda after the millennium:

Awerbuch and Berger (2003) use MVP theory to evaluate existing and projected generation mixes in the European Union in a total generation cost framework. The authors compare existing risk-return combinations to a set of efficient portfolios that minimize generation costs at a given level of market risk. In general, the results indicate that the existing and projected EU generating mixes are sub-optimal from a risk-return perspective. The analyses further suggest that portfolios with lower cost and risk can be developed by including larger amounts of renewables (which typically have high fixed but very low variable costs).

Several applications of MVP theory in electricity markets published hereafter are all based on the same key modeling characteristics with some adjustments tailored to the specific focus of analysis:

- Empirical applications focus on different regional or national markets such as Ireland (Awerbuch, 2004), the European Union at large (Awerbuch, 2006; Jansen et al., 2006), Mexico, the U.S. (Awerbuch, 2006) and California (White et al., 2007).

- Jansen et al. (2006) adopt a number of adjustments and additions with respect to the above-mentioned MVP frameworks. The authors use energy based instead of generation capacity based portfolios weights for the respective fuel. Moreover, incremental technology deployment analysis is applied.

- Besides fuel price risk, the MVP framework can be extended to incorporate other kinds of cost risks, such as operation and maintenance risk and construction time risks (Jansen et al., 2006; Awerbuch and Berger, 2003) or components cost risks.

- To derive feasible transition paths from an existing fuel mix to an optimized state, some optimization models differentiate between new and existing generation capacity (Awerbuch and Berger, 2003). 
Intended to enable policy makers and electricity supply system analysts to investigate the mix of power generation technologies, the above-mentioned applications of MVP analyses reflect a welfare perspective. According to standard microeconomic theory such a system optimum should coincide with the optimal investment decisions of individuals if the characteristics of perfect competition are fulfilled. ${ }^{4}$ Dedicated to an investor's perspective on electricity markets, Roques et al. (2006a,b, 2008) propose a model framework which aims at deriving efficient generation portfolios from the perspective of a single power producer. Therefore, the authors introduce electricity prices as another risk factor and use profit as the meaningful return measure. The derivation of the efficient frontier for the assumed generation portfolio is accomplished in a two-step model. In the first simulation step, the distribution parameters for the net present value of each generation technology are determined. The second step simulates the multivariate distribution characteristics of the different fuel types.

Since mean and variance of the different fuel prices represent exogenous key inputs for the portfolio optimization models, improved econometric estimation techniques are a further focal point in research. In contrast to most MVP studies following Awerbuch and Berger (2003), which rely on ordinary least square (OLS) regressions for econometric estimation of the covariance matrix, Humphreys and McClain (1998) introduce a time-varying covariance matrix for different fuel types derived from a GARCH (generalized autoregressive conditional heteroskedasticity) model to derive an efficient portfolio frontier of the energy mix consumed in the United States. Another refined estimation approach is suggested by Krey and Zweifel (2006) to take into account the correlation of shocks in fuel prices. The authors apply the seemingly unrelated regression estimation (SURE) method for filtering out time-invariant covariance matrices as input to find efficient generation mixes for Switzerland and the United States.

Whereas MVP theory focuses on risk, multi-criteria portfolio diversity analysis has gained some attention as a second important concept for portfolio selection. This broad framework - originally applied in biology is also applicable in an environment of uncertainty and ignorance ${ }^{5}$ : Referring to the work of Stirling (1994) which represents the first application of diversity to electricity markets, Awerbuch et al. (2006) have recently proposed an integrative approach which combines the concepts of MVP and portfolio diversity.

The cost-related studies following the work of Awerbuch and Berger (2003) however share the major common drawback that unit costs (total generation cost per MWh) are treated as constant exogenous input parameters. Yet this would only be valid if full load hours of all considered technologies were not influenced

\footnotetext{
${ }^{4}$ This proposition, also known as the first theorem of welfare economics, is described in many standard textbooks on economics and goes back to the Adam Smith's postulations of the "invisible hand". Among others, Pigou (1932), chapter II, contributed fundamentally to a precise formulation of this theorem and its preconditions. One of the first mathematical proofs was published by Lange (1942).

${ }^{5}$ Economy textbooks usually distinguish between risk (if sufficient information on the probability distribution for a range of possible outcomes exists), uncertainty (if there is no basis for the assignment of probabilities), and ignorance (if there is no knowledge about possible outcomes at all). See also Stirling (1994); Awerbuch et al. (2006).
} 
by the portfolio composition. If however MVP theory is applied market wide, the optimal technology mix can clearly affect the effective operating hours and consequently influencing the shape of the price duration curve and unit costs. Therefore, the assumption of stable distributions for unit costs, as done more or less implicitly in the above-mentioned studies, would require a stable merit order operation pattern which is especially in the long-run - not only a heroic assumption but also a severe inconsistency.

As critical as stable unit costs is the use of a stable electricity price distribution derived from historical data as done by Roques et al. (2008). This consequently implies a net present value (NPV) distribution that neglects the fact that portfolio choices will also influence electricity prices in the long run. To compass the problem of modeling technology-specific adjustments of full load hours and implications on the electricity price distribution, the authors explicitly restrict their profit-oriented model framework to base-load portfolios in which all technologies are assumed to operate at the same full load hours. Although this assumption and the limitation to base load portfolios avoids inconsistencies in the modeling results, it however prevents to derive conclusions about the long-term market equilibrium.

For conclusions on the optimal generation technology mix for an electricity market as a whole, a solid long-term modeling framework should therefore better be based on the integrated modeling of the long-term market optimum taking into account operating and investment costs instead of unit costs: Based on this modeling principle, Gotham et al. (2009) proposed more recently a static cost-based model for optimal capacity allocation in a mean-variance framework with different load segments to be served. In addition, the model presented by Delarue et al. (2009) captures ramp-up costs as well as uncertain availability of renewable technologies such as wind.

It is remarkable that the studies on MVP optimization in electricity markets published so far utilize predominantly simulation techniques or numerical solvers to derive the optimal generation fuel mix in the respective problem formulation. It was thus very tempting to analytically study optimality conditions for mean-variance portfolios in electricity generation systems derived from a model applicable to continuous load functions.

\subsection{Measuring risk and return}

The question of the most appropriate risk measure is closely interlinked with the respective measure of return and is assumably as old as the question of the risk-minimal portfolio itself.

As proposed by Markowitz $(1952,1959)$, most classic MVP applications to financial markets use expected holding period return (HPR) as expected portfolio return measure. Markowitz originally used variance of returns as the corresponding measure of dispersion and showed that variance-related measures such as standard deviation or coefficient of variation lead to the same set of efficient portfolios.

In competitive electricity markets with deterministic and price inelastic demand, maximization of expected welfare is equivalent with minimization of expected total generation cost, which is well-known as the 
peak-load pricing problem. ${ }^{6}$ To adapt the MVP theory from financial markets to electricity portfolios from an economic-wide perspective, Bar-Lev and Katz (1976); Humphreys and McClain (1998); Awerbuch and Berger (2003) and White et al. (2007) propose to maximize the inverse of the weighted average portfolio costs (in MWh per monetary unit) as "return" measure based on this rationale. In analogy to the HPR concept for financial portfolios the authors present risk as a percentage rate defined as the (annualized) standard deviation of intra-period cost changes. This allows to keep many aspects of Markowitz' MVP framework such as the efficient frontier depiction, but involves at the same time avoidable inconsistencies: As pointed out by Jansen et al. (2006), maximizing the inverse of costs and minimizing costs do in general not lead to the same results. By deploying simple costs and the corresponding variance in their proposed framework, e.g. Jansen et al. (2006) and Gotham et al. (2009) avoid inconsistencies in the risk and return measure without compromising the clarity of the classical MVP theory.

In contrast, Krey and Zweifel (2006) propose a MVP framework where the objective is to minimize (negative) "return" which the authors define as the rate of increase per unit of electricity-generating cost. Risk is defined as the corresponding standard deviation. Not surprising, this risk-return measure leads to biased and counter-intuitive results: According to Krey and Zweifel (2006), solar would be the only selected technology in a risk neutral economy in which the sole interest were to maximize expected welfare (i.e. to minimize the expected increase of electricity-generation costs). ${ }^{7}$

Aware of the described weaknesses of both variance or standard deviation of return and HPR risk as risk measures Lemming and Meibom (2003), Kleindorfer and Li (2005), and Balagopal and Gilliland (2005) propose frameworks to adopt MVP theory to the concept of value-at-risk. However, it has to be remarked that both variance and VaR are non-coherent risk measures and therefore comprise disadvantages.

The biggest benefit of applying variance for risk quantification is the clarity of the classical MVP framework. This is the reason why we decided to stick to this approach in this paper. A comparison of variance with different downside risk measures (e.g. lower partial moments, conditional value-at-risk) in electricity portfolio applications could be an interesting and yet missing building block in this area of research.

\footnotetext{
${ }^{6}$ The peak-load pricing theory goes back to the work of Boiteux (1960). A general description of the peak load pricing problem in the deterministic case as well as more comprehensive extensions for stochastic fuel prices and demand fluctuations can be found e.g. in Crew and Kleindorfer (1986), Oren (2000), Fischer and Serra (2003), Weber and Swider (2004), Weber (2005).

${ }^{7}$ Krey and Zweifel (2006) describe only vaguely the motives of their peculiar return definition: "In the case of both Switzerland and the United States, who are net importers, power constitutes a liability rather than an asset since payments must be made to foreign suppliers. The (negative) rate of return on the power portfolio then becomes the rate of increase of the energy bill - which now is to be minimized rather than maximized." In analogy, this would however mean that a consumer does not select the cheapest retailer to optimize his electricity bill, but that one with the least price increase (in past years). This selection can however be far away from the cost-minimal solution.
} 


\section{Modeling optimal investment policies with portfolio risks in electricity markets}

\subsection{Deterministic peak-load pricing problem}

The general peak-load pricing problem which leads to a long-term cost-efficient generation system can be formulated as a static optimization problem (cf. e.g. Crew and Kleindorfer, 1986) as shown in Eqs. (1) to (4): We consider an electricity system with $u \in\{1, \ldots, n\}$ plant technologies available. From a societal perspective, the objective is to minimize the sum of total operating costs, $C_{o p, u}$, plus annualized capacity investment costs, $C_{i n v, u}$, summed over the available technologies $u$ and over the total planning period $[0 ; T]$ (e.g. a year). Let the latter be broken down into time steps of equal length $t \in[0 ; T]$ (e.g. hours). Eqn. (4) represents the demand constraint with $D:[0 ; T] \rightarrow \mathbb{R}_{+}, t \mapsto D(t)$ denoting the total system demand function. The demand to be supplied is assumed to be price inelastic which can be considered as a simplifying but within a wide range of operating costs fairly realistic assumption. ${ }^{8}$ The capacity constraint which assures that the output of each plant, $y_{u, t}$, is less than or equal its capacity, $K_{u}$, is given in Eqn. (3):

$$
\begin{aligned}
C^{*}= & \min _{y_{u, t}, K_{u}} C\left(y_{u, t}, K_{u}\right) \\
\text { s.t. } C= & \int_{0}^{T} \sum_{u} C_{o p, u} \mathrm{~d} t+\sum_{u} C_{i n v, u}=\int_{0}^{T} \sum_{u} y_{u, t} \cdot c_{o p, u, t} \mathrm{~d} t+\sum_{u} K_{u} \cdot c_{i n v, u} \\
& y_{u, t}-K_{u} \leq 0 \quad \forall \quad t, u \\
& \sum_{u} y_{u, t} \geq D(t) \quad \forall \quad t
\end{aligned}
$$

Operating costs at time $t$ are a function $C_{o p, u}\left(c_{o p, u, t}, y_{u, t}\right)$ of specific operating costs $c_{o p, u, t}(€ / \mathrm{MWh})$ and the instantaneous output level $y_{u, t}(\mathrm{MW})$. In addition, we will write the investment costs in the following sections as $C_{i n v, u}\left(K_{u}, c_{i n v, u}\right)$, indicating the dependency on the installed capacity $K_{u}$ and the specific investment $\operatorname{costs} c_{i n v, u}\left(€ / \mathrm{MW}_{e l}\right)$. Therefore, the plant capacities $K_{u}$ and the corresponding output levels $y_{u, t}$ are the decision variables to be optimized.

To allow a better understanding of the results, we assume in this model formulation full capital flexibility which can realistically only be assumed over a very long planning horizon. ${ }^{9}$ Furthermore, we neglect plant indivisibilities and other technology-specific constraints not reflected in the average operating costs such as ramp-up costs and times. ${ }^{10}$ However, these assumptions can easily be implemented in any numerical large-scale model setup and are not in focus of this analysis.

\footnotetext{
${ }^{8}$ An extension of the deterministic peak-load pricing problem considering the effect of price elastic demand can e.g. be found in Oren (2000)

${ }^{9}$ In a differentiated modeling of the peak-load pricing problem regarding new and historic investments as e.g. done by Gotham et al. (2009), historic (sunk) investment costs are neglected. This enables the model to capture the optimal transition from an existing plan fleet the future long-term optimum, which is however not the main purpose of our analysis.

${ }^{10}$ See e.g. Fischer and Serra (2003) for an extended peak-load pricing model with plant indivisibilities.
} 


\subsection{Risk-adjusted investment optimum with uncertain fuel prices}

To derive efficient frontiers of asset combinations, classical MVP theory assumes that investors' portfolio preferences depend solely on mean and variance of the expected return. The portfolio with the smaller variance of return at the same level of expected return or the portfolio with the higher expected return at the same level of return variance will be preferred.

The $\left(\mu, \sigma^{2}\right)$ decision principle to be consistent with maximization of expected utility in rational decision making under uncertainty ${ }^{11}$ requires either investors to act based on quadratic utility functions or returns to be normally distributed and investors to behave risk aversely. ${ }^{12}$

Frequently used in optimization literature are preference functions of the form $\Psi(a):=\mathbb{E}[X(a)]-$ $\frac{A}{2} \operatorname{Var}[X(a)]$, where $a$ denotes a decision alternative and $X$ the corresponding random payoff. Schneeweiss (1965) has shown that for normally distributed payoffs, exponential utilities with constant absolute risk aversion are necessary and sufficient for consistency of the preference $\Psi\left(\mu, \sigma^{2}, a\right)$ with the rational principle of expected utility maximization.

Transferring this approach to the generation portfolio risks induced by fuel price uncertainty, we can use the proposed preference function to extend the objective function of the standard peak-load pricing model by a variance term that captures the risk from uncertain operating costs due to fluctuations in primary energy prices. Assuming societal preferences being described by the preferences of a representative consumer with an exponential utility function of the form $U(x)=-\exp (-A x)$, the expected dis-utility can then be approximated by the following $\left(\mu, \sigma^{2}\right)$ preference as a function of the expected generation costs and the corresponding variance: ${ }^{13}$

$$
L=\mathbb{E}[C]+\frac{1}{2} A \cdot \operatorname{Var}[C],
$$

The parameter $A$ denotes the society's risk attitude and reflects for $A=0$ risk neutrality, $A>0$ risk aversion and $A<0$ risk proclivity.

The proposed model focuses on input price risks of electricity generation. Most important, fuel price fluctuations can financially affect generation costs - in principle both in the long-term and in the short-term. Unlike short-term risks, which can be hedged on energy forward markets, long-term fuel price uncertainties remain as a major risk factor. Therefore, we conceive the optimal generation portfolio selection problem as a two-stage problem. At the first stage, investment is carried out, i. e. capacities are selected based on known investment cost and uncertain fuel cost. The second stage covers the power plant operation over a representative period, i. e. a typical year. At this stage, the actual fuel prices are revealed. Fuel price fluctuations within the operating period are disregarded in this article, assuming that those may be

\footnotetext{
${ }^{11}$ In economic textbooks frequently referred to as "Bernoulli's principle".

${ }^{12}$ See e.g. Tobin (1958).

${ }^{13}$ Further assumptions are normally distributed fuel prices, additively separable utilities between electricity and other goods and no hedging possibilities of electricity fuel price risk outside the electricity sector. .
} 
eliminated through hedging. Non market risks, e.g. operational or technical risk factors such as availability or construction cost risks, are also not considered in the model. To capture the long-term fuel price fluctuations, specific operating costs of each technology $u$ are modeled as random variables ${ }^{14}$ with obtained realizations being taken as constant throughout the operating period $[0 ; T] \cdot{ }^{15}$ Expected operating costs are denoted by $\bar{c}_{o p, u}:=\mathbb{E}\left[\tilde{c}_{o p, u}\right]$. The covariance in specific operation costs of plants $u$ and $v$ is denoted by the matrix $\sigma_{u v}$, i.e. $\tilde{c}_{o p, u}$ are $u$-variate jointly distributed. For a shorter notation, we denote $Q_{u}$ the energy produced by technology $u$ in the period $[0 ; T]$, i.e. $Q_{u}:=\sum_{t} y_{u, t}$. Then, as shown in appendix B.1, the expected dis-utility capturing expected total generation costs and (fuel) cost risk can be specified as

$$
L=\sum_{u} Q_{u} \bar{c}_{o p, u}+\sum_{u} C_{i n v, u}+\frac{A}{2}\left(\sum_{u} \sigma_{u}^{2} Q_{u}^{2}+\sum_{u} \sum_{v, v \neq u} \sigma_{u v} Q_{u} Q_{v}\right) .
$$

Without loss of generality, demand is assumed to be given in a decreasing order (i.e. rearranged in form of the load duration curve) by the function $D:[0 ; T] \rightarrow \mathbb{R}_{+}, t \mapsto D(t)$ which we assume to be strictly monotone with $D(0)=D_{\max }$. Furthermore, we order $n$ technologies by increasing operating costs, i.e. $\forall u, v \in\{1, \ldots, n\},\left(\bar{c}_{o p, u}<\bar{c}_{o p, v}\right)$. We exclude the possibility of reversals in the merit order such that no realization of operating costs with $c_{o p, u} \geq c_{o p, v}$ can occur. ${ }^{16}$ Then the second-stage problem of optimal operation is solved as in the conventional peak-load-pricing problem based on the merit order. For the technology with the lowest operating costs, i. e. technology 1, the upper bound of operating duration is always $t_{0}=T$. The lower bound is given through $D\left(t_{1}\right)=K_{1}$, since technology one will run at full capacity as soon as demand exceeds capacity $K_{1}$. Similarly for technology two, the upper bound for operation hours is given by $D\left(t_{1}\right)=K_{1}$, and the lower by $D\left(t_{2}\right)=K_{1}+K_{2}$ and so forth (see Figure 1). Finally, it can be seen that the lower bound of the operating time of the $n$-th technology is zero, i.e. $t_{n}=0$. By introducing the cumulative capacity $K_{u}^{c}=\sum_{j=1}^{u} K_{j}$, and defining $R(K)$ as the inverse of the monotonously decreasing function $D(t)$, we may write $t_{u}=R\left(K_{u}^{c}\right)$. Now, solving the first-stage portfolio selection problem is equivalent to determining the cumulative capacities $K_{u}^{c}$. We additionally define the integral to the inverse demand function

$$
Q^{I}\left(K_{u}^{c}\right)=\int_{0}^{K_{u}^{c}} R(\kappa) \mathrm{d} \kappa
$$

The optimization problem may now be reformulated, using only $K_{u}^{c}$ as decision variables. In timecontinuous notation, this yields

\footnotetext{
${ }^{14}$ Throughout this article, random variables are indicated by a " $"$, whereas their realizations are written as plain letters.

${ }^{15}$ Because operating costs are constant within the planning period, we write $c_{o p, u}$ instead of $c_{o p, u, t}$.

${ }^{16}$ This simplification can be justified because the empirically estimated year-to-year risk for reversals in the merit order is less than $1 \%$ for all considered technologies and hence extremely low (cf. Section 4 ).
} 


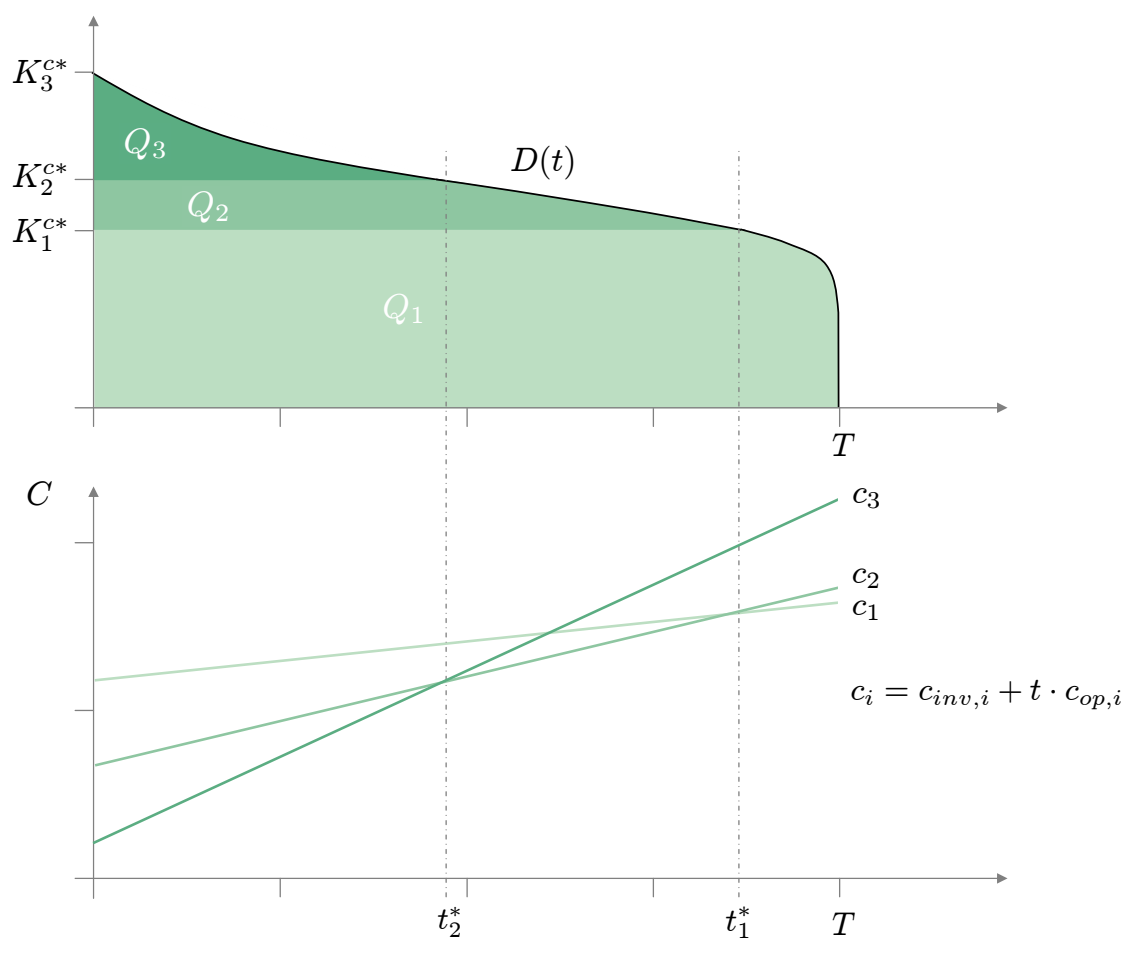

Figure 1: Graphical solution of the deterministic peak-load pricing problem from load duration curve and full-cost curves

$$
\begin{aligned}
L^{*}= & \min _{K_{u}^{c}} L \\
\text { s.t. } & L=\sum_{u=1}^{n} c_{i n v, u}\left(K_{u}^{c}-K_{u-1}^{c}\right)+\bar{c}_{o p, u} Q_{u}+\frac{A}{2}\left(\sum_{u=1}^{n} \sum_{v=1}^{n} \sigma_{u v} Q_{u} Q_{v}\right) \\
& K_{u}^{c}-K_{u-1}^{c} \geq 0 \quad(1 \leq u \leq n) \\
& Q_{E} \leq Q^{I}\left(K_{n}^{c}\right)
\end{aligned}
$$

with

$$
Q_{u}=Q^{I}\left(K_{u}^{c}\right)-Q^{I}\left(K_{u-1}^{c}\right) .
$$

and $Q_{E}$ denoting total energy demand over the considered period,

$$
Q_{E}=\int_{0}^{T} D(t) \mathrm{d} t
$$

The corresponding Lagrangian writes:

$$
\begin{array}{r}
\mathcal{L}_{n}=\sum_{u=1}^{n} c_{i n v, u}\left(K_{u}^{c}-K_{u-1}^{c}\right)+\bar{c}_{o p, u} Q_{u} \\
+\frac{A}{2}\left(\sum_{u=1}^{n} \sum_{v=1}^{n} \sigma_{u v} Q_{u} Q_{v}\right)+\sum_{u=1}^{n} \mu_{u}\left(K_{u-1}^{c}-K_{u}^{c}\right)+\lambda\left(Q_{E}-Q^{I}\left(K_{n}^{c}\right)\right)
\end{array}
$$


Before exploring this general optimization problem in more detail, two specific configurations are investigated, contained as extremes in the general portfolio problem.

\subsection{Standard solution for pure cost efficient portfolios with $n$ technologies}

One limiting case obviously embedded in the general formulation is the deterministic peak-load pricing problem, corresponding to $A=0$. The other case to be looked at is the pure variance-minimizing problem, to which the general problem converges as $A \rightarrow+\infty$. As shown in Crew and Kleindorfer (1986), the deterministic peak-load pricing problem with $A=0$ and $n$ technologies may also be solved graphically using the load duration curve and the full-cost curves of the respective technologies (see Figure 1). Formally, the Karush-Kuhn-Tucker (KKT) conditions corresponding to the Lagrangian (6) are here:

$$
\begin{array}{llll}
\frac{\partial \mathcal{L}_{n}}{\partial K_{u}^{c}}=c_{i n v, u}-c_{i n v, u+1}+\left(\bar{c}_{o p, u}-\bar{c}_{o p, u+1}\right) t_{u}-\mu_{u}+\mu_{u+1} \geq 0, & \perp & K_{u}^{c} \geq 0 & \text { with }(1 \leq u \leq n-1) \\
\frac{\partial \mathcal{L}_{n}}{\partial K_{n}^{c}}=c_{i n v, n}+\bar{c}_{o p, u} t_{n}-\mu_{n}-\lambda t_{n} \geq 0, & & K_{n}^{c} \geq 0 & \\
\frac{\partial \mathcal{L}_{n}}{\partial \mu_{u}}=K_{u-1}^{c}-K_{u}^{c} \leq 0, & & & \mu_{u} \geq 0 \\
\frac{\partial \mathcal{L}_{n}}{\partial \lambda}=Q_{E}-Q^{I}\left(K_{n}^{c}\right) \leq 0, & \text { with }(1 \leq u \leq n)
\end{array}
$$

Thereby $K_{0}^{c}=0$ is used as additional convention. Obviously the last set of conditions pushes $K_{n}^{c}$ to be at least equal to $D(0)$, so that all energy is provided by the total capacity installed and consequently even the peak-load demand is covered. Simultaneously, (14) then implies that

$$
\lambda=\bar{c}_{o p, u}+\frac{c_{i n v, n}-\mu_{n}}{t_{n}}
$$

Given that $t_{n}=R\left(K_{n}^{c}\right)=0$ follows from $K_{n}^{c}=D(0)$, the last equation implies $\lambda \rightarrow+\infty$. This at first sight awkward result reflects the well-known property that in the peak-load-pricing model the shadow price in the (here infinitesimal) peak-load moment covers the full investment costs of the peak technology. From (13) optimal lower bounds of operating hours may be written

$$
t_{u}=\frac{c_{i n v, u}-c_{i n v, u+1}-\mu_{u}+\mu_{u+1}}{c_{o p, u+1}-c_{o p, u}},(1 \leq u<n),
$$

If $K_{u}>0$ is assumed throughout, (15) implies that all $\mu_{u}$ are equal to zero. Consequently (18) may be directly used to compute capacities

$$
\begin{array}{r}
t_{u}^{o}=\frac{c_{i n v, u}-c_{i n v, u+1}}{c_{o p, u+1}-c_{o p, u}},(1 \leq u<n), \\
K_{u}^{c *}=D\left(t_{u}^{o}\right), K_{u}^{*}=K_{u}^{c *}-K_{u-1}^{c *},(1 \leq u \leq n) .
\end{array}
$$

In fact the $t_{u}^{o}$ may be used generally to check the validity of the assumption $K_{u}>0$. As shown in Appendix B.2, $K_{u}^{*}>0$ only if $t_{u}^{o}<t_{u-1}^{o}$. Stated in other words, technology $u$ is not part of the cost-efficient portfolio if $t_{u}^{o} \geq t_{u-1}^{o}$. A graphical interpretation and further implications of this finding are discussed in Appendix B.2. 


\subsection{Standard solution for pure variance efficient portfolios with $n$ technologies}

For the second limiting case with $A \rightarrow+\infty$, the Lagrangian (6) may be rewritten as:

$$
\mathcal{L}_{n}=\sum_{u=1}^{n} \sum_{v=1}^{n} \sigma_{u v} Q_{u} Q_{v}-\sum_{u=1}^{n} \mu_{u}^{Q} Q_{u}+\lambda^{Q}\left(Q_{E}-\sum_{u=1}^{n} Q_{u}\right)
$$

Thereby the $Q_{u}$ are directly used as decision variables, since this allows a more convenient treatment. Yet through $Q_{u}=Q^{I}\left(K_{u}^{c}\right)-Q^{I}\left(K_{u-1}^{c}\right)$ a unique mapping between the $Q_{u}$ 's and $K_{u}^{c}$ 's is established, which may be later used to transform results. The KKT-conditions are here:

$$
\begin{aligned}
& \frac{\partial \mathcal{L}_{n}}{\partial Q_{u}}=\sum_{v=1}^{n} \sigma_{u v} Q_{v}-\mu_{u}^{Q}-\lambda^{Q} \geq 0, \quad \quad \perp \quad Q_{u} \geq 0 \quad \text { with }(1 \leq u \leq n) \\
& \frac{\partial \mathcal{L}_{n}}{\partial \mu_{u}^{Q}}=-Q_{u} \leq 0, \quad \perp \quad \mu_{u}^{Q} \geq 0 \quad \text { with }(1 \leq u \leq n) \\
& \frac{\partial \mathcal{L}_{n}}{\partial \lambda^{Q}}=Q_{E}-\sum_{u=1}^{n} Q_{u} \leq 0 \quad \perp \quad \lambda^{Q} \geq 0
\end{aligned}
$$

A matrix notation is advantageous for the further treatment, hence the key conditions are rewritten with $\mathbf{i}^{T}=(1, \ldots, 1)^{T}$ denoting the $n$-dimensional one vector:

$$
\begin{aligned}
\boldsymbol{\Sigma} \mathbf{Q}-\boldsymbol{\mu}^{Q}-\lambda^{Q} \mathbf{i} & =\mathbf{0} \\
Q_{E}-\mathbf{i}^{T} \mathbf{Q} & =0
\end{aligned}
$$

In fact the case $Q_{u}=0$ does not immediately lead to a determinate value for $\mu_{u}^{Q}$ since each $\mu_{u}^{Q}$ only appears in one inequality (22). Therefore we assume without loss of generality the left part of (22) to be fulfilled with equality. For the left part of the last condition also an equality assumption is save, since the opposite would imply that in a cost minimization framework excess quantities were available for free.

Obviously two cases have to be distinguished for the determination of the variance-minimal portfolio: (1) The assets in the portfolio are linearly independent. (2) The assets are linearly dependent. In the latter case, at least the stochastic variation of one asset may be replicated by a combination of the others. Then obviously the variance minimizing portfolio may also be not unique and without further restrictions also a non-trivial risk free portfolio may be constructed. This case is therefore not further considered here. In the first case by contrast the covariance matrix will be not only positive semi-definite ${ }^{17}$ but strictly positive definite. Consequently it is also invertible and the two equations (22) and (24) may then be combined to yield unique solutions for $\lambda^{Q}$ and $\mathbf{Q}$ as a function of $\mu^{\mathbf{Q}}$ (cf. Appendix B.3):

$$
\begin{aligned}
\lambda^{Q} & =\frac{1}{\mathbf{i}^{T} \boldsymbol{\Sigma}^{-\mathbf{1}} \mathbf{i}}\left(Q_{E}-\mathbf{i}^{T} \boldsymbol{\Sigma}^{-\mathbf{1}} \boldsymbol{\mu}^{Q}\right) \\
\mathbf{Q} & =\boldsymbol{\Sigma}^{-\mathbf{1}}\left(\lambda^{Q} \mathbf{i}+\boldsymbol{\mu}^{Q}\right)=\frac{Q_{E}}{\mathbf{i}^{T} \boldsymbol{\Sigma}^{-\mathbf{1}} \mathbf{i}} \boldsymbol{\Sigma}^{-\mathbf{1}} \mathbf{i}+\left(\boldsymbol{\Sigma}^{-\mathbf{1}}-\frac{1}{\mathbf{i}^{T} \boldsymbol{\Sigma}^{-\mathbf{1}} \mathbf{i}} \boldsymbol{\Sigma}^{-\mathbf{1}} \mathbf{i i}^{T} \boldsymbol{\Sigma}^{-\mathbf{1}}\right) \boldsymbol{\mu}^{Q}
\end{aligned}
$$

\footnotetext{
${ }^{17}$ This is the case for any covariance matrix, cf. Horn and Johnson (1985), p. 392.
} 
In this case the optimization problem is strictly convex and again a straight-forward procedure may be used to check whether all technologies $u$ are included in the risk-minimal portfolio. One has merely to compute the vector $\mathbf{Q}^{o}$ :

$$
\mathbf{Q}^{o}=\frac{Q_{E}}{\mathbf{i}^{T} \boldsymbol{\Sigma}^{-\mathbf{1}} \mathbf{i}} \boldsymbol{\Sigma}^{-\mathbf{1}} \mathbf{i}
$$

It holds $Q_{u}>0$ for all $1 \leq u<n$ if and only if $Q_{u}^{o}>0$ for all $1 \leq u<n$, as shown in appendix B.3. Consequently, in this case the variance-minimal solution is given by $\mathbf{Q}^{\mathbf{r} *}=\mathbf{Q}^{\mathbf{o}}$, where the element $Q_{u}^{o}$ corresponds to:

$$
Q_{u}^{o}=\frac{Q_{E}}{\mathbf{i}^{T} \boldsymbol{\Sigma}^{-\mathbf{1}} \mathbf{i}} \sum_{v=1}^{n}\left\{\boldsymbol{\Sigma}^{-\mathbf{1}}\right\}_{u v}
$$

In other words, only if the row-sums of the inverse covariance matrix $\boldsymbol{\Sigma}^{-1}$ are all positive, all available technologies will be part of the variance-minimal portfolio. Then, the optimal amount of energy produced by technology $u$ is obtained as weighted share of the total energy produced, where the $u$-th row sum of the inverted covariance matrix $\boldsymbol{\Sigma}^{-1}$ is used as weighting factor. Remark that for instance $\boldsymbol{\Sigma}^{-\mathbf{1}}$ being strictly diagonally dominant with positive diagonal entries can thus guarantee that all technologies are part of the risk-minimal portfolio. ${ }^{18}$ Furthermore it can be shown that the variance minimization problem has a unique solution if $\boldsymbol{\Sigma}$ is positive definite (cf. appendix B.3).

\subsection{Standard solutions to the combined portfolio problem with $n$ technologies}

The optimal portfolio in the general case of combined cost-risk optimization may in principle be derived using a combination of the two previously shown approaches. This is however complicated by differences in notation and differences in solution logics between the two cases. The risk-minimization case requires matrix-inversion, therefore introducing matrix-notation is also necessary for the cost-minimization part. This can be achieved by introducing the lag-operator in matrix form $\mathbf{L}$ through:

$$
\mathbf{L}=\left(\begin{array}{ccccc}
0 & 0 & \cdots & 0 & 0 \\
1 & 0 & \cdots & 0 & 0 \\
0 & 1 & \cdots & 0 & 0 \\
\vdots & \vdots & \ddots & \vdots & \vdots \\
0 & 0 & \cdots & 1 & 0
\end{array}\right)
$$

Then the Lagrangian may be written

$$
\begin{aligned}
\mathcal{L}_{n}= & \mathbf{c}_{\mathbf{i n v}}{ }^{T}(\mathbf{I}-\mathbf{L}) \mathbf{K}^{\mathbf{c}}+\overline{\mathbf{c}}_{\mathbf{o p}}^{T}(\mathbf{I}-\mathbf{L}) Q^{I}\left(\mathbf{K}^{\mathbf{c}}\right)+\frac{A}{2}\left((\mathbf{I}-\mathbf{L}) Q^{I}\left(\mathbf{K}^{\mathbf{c}}\right)\right)^{T} \boldsymbol{\Sigma}(\mathbf{I}-\mathbf{L}) Q^{I}\left(\mathbf{K}^{\mathbf{c}}\right)+ \\
& +\boldsymbol{\mu}^{T}(\mathbf{I}-\mathbf{L}) \mathbf{K}^{\mathbf{c}}+\lambda\left(Q_{E}-Q^{I}\left(K_{n}^{c}\right)\right)
\end{aligned}
$$

\footnotetext{
${ }^{18}$ A Hermitian, strictly diagonally dominant matrix with positive diagonal entries is also positive definite (cf. Horn and Johnson, 1985, Corollary 7.2.3). However, the converse cannot be concluded in general.
} 
Denoting $\frac{\partial \mathcal{L}_{n}}{\partial \mathbf{K}^{c}}=\left(\frac{\partial \mathcal{L}_{n}}{\partial K_{1}^{c}}, \ldots, \frac{\partial \mathcal{L}_{n}}{\partial K_{n}^{c}}\right)^{T}$ and $\frac{\partial \mathcal{L}_{n}}{\partial \boldsymbol{\mu}}=\left(\frac{\partial \mathcal{L}_{n}}{\partial \mu_{1}}, \ldots, \frac{\partial \mathcal{L}_{n}}{\partial \mu_{n}}\right)^{T}$, the corresponding KKT conditions can be derived using matrix calculus:

$$
\begin{array}{rlrl}
\frac{\partial \mathcal{L}_{n}}{\partial \mathbf{K}^{\mathbf{c}}}=(\mathbf{I}-\mathbf{L})^{T} \mathbf{c}_{\mathbf{i n v}}+\operatorname{diag}\left(t\left(\mathbf{K}^{\mathbf{c}}\right)\right)(\mathbf{I}-\mathbf{L})^{T} \mathbf{c}_{\mathbf{o p}}+(\mathbf{I}-\mathbf{L})^{T} \boldsymbol{\mu}+\lambda\left(\mathbf{i}_{\mathbf{n}}\right)^{T} t\left(\mathbf{K}^{\mathbf{c}}\right)+ & & \\
\quad+A \operatorname{diag}\left((\mathbf{I}-\mathbf{L}) t\left(\mathbf{K}^{\mathbf{c}}\right)\right) \mathbf{\Sigma}(\mathbf{I}-\mathbf{L}) Q^{I}\left(\mathbf{K}^{\mathbf{c}}\right) \geq \mathbf{0}, & \perp & \mathbf{K}^{\mathbf{c}} \geq \mathbf{0}, \\
\frac{\partial \mathcal{L}_{n}}{\partial \boldsymbol{\mu}}=(\mathbf{I}-\mathbf{L}) \mathbf{K}^{\mathbf{c}} \leq \mathbf{0}, & \perp & \boldsymbol{\mu} \geq \mathbf{0}, \\
\frac{\partial \mathcal{L}_{n}}{\partial \lambda}= & Q_{E}-Q^{I}\left(K_{n}^{c}\right) \leq 0, & \perp & \lambda \geq 0 .
\end{array}
$$

Similar to the previous problem, we can again assume $Q_{E}=Q^{I}\left(K_{n}^{c}\right)$ and condition (31) to be fulfilled with equality. Hence, $\frac{\partial \mathcal{L}_{n}}{\partial \mathbf{K}^{\mathrm{c}}}=\mathbf{0}$ is the remaining central optimality condition to be solved. From $K_{n}^{c}=D(0)$, it is however clear that $t_{n}=0$ and consequently $\lambda$ is eliminated from the optimality condition. Shifting the risk-free term and the risk term to the two sides of the equation, we can now rewrite Eqn. (31) as shown in Appendix B.4:

$$
-A \boldsymbol{\Sigma} Q\left(\mathbf{K}^{\mathbf{c}}, \mathbf{L} \mathbf{K}^{\mathbf{c}}\right)=\operatorname{diag}\left((\mathbf{I}-\mathbf{L}) t\left(\mathbf{K}^{\mathbf{c}}\right)\right)^{-1}\left((\mathbf{I}-\mathbf{L})^{T}\left(\mathbf{c}_{\mathbf{i n v}}+\boldsymbol{\mu}\right)+\operatorname{diag}\left(t\left(\mathbf{K}^{\mathbf{c}}\right)\right)(\mathbf{I}-\mathbf{L})^{T} \mathbf{c}_{\mathbf{o p}}\right)
$$

Focusing on solutions which include all technologies, we assume again $\boldsymbol{\mu}=\mathbf{0}$. At a closer look, optimality condition (34) represents an $n$-dimensional equation system consisting of two functional terms $\mathbf{l}, \mathbf{r}$ of the form

$$
\left(\begin{array}{c}
l_{1}\left(Q_{1}\left(K_{1}^{c}\right), \ldots, Q_{n}\left(K_{n}^{c}, K_{n-1}^{c}\right)\right) \\
l_{2}\left(Q_{1}\left(K_{1}^{c}\right), \ldots, Q_{n}\left(K_{n}^{c}, K_{n-1}^{c}\right)\right) \\
\vdots \\
l_{n}\left(Q_{1}\left(K_{1}^{c}\right), \ldots, Q_{n}\left(K_{n}^{c}, K_{n-1}^{c}\right)\right)
\end{array}\right)=\left(\begin{array}{c}
r_{1}\left(t_{1}\left(K_{1}^{c}\right)\right) \\
r_{2}\left(t_{2}\left(K_{2}^{c}\right), t_{1}\left(K_{1}^{c}\right)\right) \\
\vdots \\
r_{n}\left(t_{n}\left(K_{n}^{c}\right), t_{n-1}\left(K_{n-1}^{c}\right)\right)
\end{array}\right)
$$

Assuming $c_{i n v, u}>c_{i n v, u+1}$ for all $u=1, \ldots, n-1$, the existence of a $\mathbf{K}^{\mathbf{c}}$ satisfying optimality condition (35) is clearly given, because row $u$ of $\mathbf{r}$ is monotone decreasing in $t_{u}$, while row $u$ of $\mathbf{l}$ is monotone increasing in $t_{u}$ (respectively in $K_{u}^{c}$ ). Indeed, it can be easily verified that for $A>0$ and $c_{i n v, u}>c_{i n v, u+1}$ for all $u=1, \ldots, n-1$ also the combined portfolio problem (8)-(11) is convex for an arbitrary covariance matrix $\boldsymbol{\Sigma}$ when taking $\mathbf{Q}$ as decision variable (cf. Appendix B.4). Furthermore, strict convexity is given if $\boldsymbol{\Sigma}$ is positive definite. Hence, any local minimum satisfying (35) must be the global minimum of the portfolio problem. Therewith, we have shown that there exists a unique solution to the risk-adjusted peak-load pricing problem under a strictly monotone load duration function with uncertain fuel prices.

Deeper insights may be gathered from the solution in the two-technology case. Therefore the following paragraph is devoted to this special case. 


\subsection{Results in the two-technology case}

In this example we consider two competitive ${ }^{19}$ generation technologies (i.e. $u=\{1,2\}$ ) with specific operating and investment costs being available to meet demand. For this case with $n=2$, the central condition to be satisfied for an interior solution with $K_{1}, K_{2}>0$ can be obtained from $\frac{\mathrm{d} \mathcal{L}_{2}}{\mathrm{~d} K_{1}}=0$ as

$$
\left(\bar{c}_{o p, 1}-\bar{c}_{o p, 2}\right) t_{1}+c_{i n v, 1}-c_{i n v, 2}+t_{1} A\left(\sigma_{1}^{2} Q_{1}-\sigma_{2}^{2} Q_{2}+\sigma_{12}\left(Q_{2}-Q_{1}\right)\right)=0
$$

Eliminating $Q_{1}$ through $Q_{1}=Q_{E}-Q_{2}$, this condition may be rewritten leading to

$$
A\left(\left(\sigma_{1}^{2}-\sigma_{12}\right) Q_{E}-\left(\sigma_{1}^{2}+\sigma_{2}^{2}-2 \sigma_{12}\right) Q_{2}\right)=\frac{c_{i n v, 2}-c_{i n v, 1}}{t_{1}}-\bar{c}_{o p, 1}+\bar{c}_{o p, 2},
$$

with

$$
Q_{2}=\int_{0}^{t_{1}}\left(D(t)-D\left(t_{1}\right)\right) \mathrm{d} t
$$

Using $t_{1}$ as decision variable, we show in Appendix B.5 that this equation is uniquely solvable for

$$
A Q_{E}\left(\sigma_{2}^{2}-\sigma_{12}\right) \geq \frac{1}{T}\left(c_{i n v, 1}-c_{i n v, 2}\right)+\bar{c}_{o p, 1}-\bar{c}_{o p, 2}
$$

As a first optimality property, two limiting parameter configurations can be observed from Eqn. (36) for the case of pure variance-efficient portfolios (i.e. $A \rightarrow+\infty$ ):

Property 3.6.1. The variance-efficient portfolio does not include technology 2 for $\frac{\sigma_{1}}{\sigma_{2}}<\rho$, while it includes only technology 2 for $\frac{\sigma_{2}}{\sigma_{1}}<\rho$, i.e.

$$
Q_{2}= \begin{cases}0 & \text { for } \frac{\sigma_{1}}{\sigma_{2}} \leq \rho \\ Q_{E} & \text { for } \frac{\sigma_{2}}{\sigma_{1}} \leq \rho\end{cases}
$$

Since per definition $0 \leq|\rho| \leq 1$, we can further conclude that technology 2 will always be included in variance-efficient portfolios for $\sigma_{1} \geq \sigma_{2}$ while technology 1 will be included in any variance-efficient portfolio for $\sigma_{2}>\sigma_{1}$ for arbitrary levels of correlation. ${ }^{20}$

\footnotetext{
${ }^{19}$ Two generation technologies $u, v$ are competitive in the long-term equilibrium, if $\left(c_{i n v, u}>c_{i n v, v}\right) \wedge\left(c_{o p, u}<c_{o p, v}\right)$. Otherwise, one technology dominates the other on a pure cost base.

${ }^{20}$ Note that the parameter ranges derived in Property 3.6.1 are consistent with those derived in Section 3.4 for the varianceminimal portfolio: First, the inverted covariance matrix $\boldsymbol{\Sigma}^{-1}$ for the case with two technologies is computed from its adjoint (cf. Horn and Johnson, 1985, p. 21) as:

$$
\boldsymbol{\Sigma}^{-1}=\frac{1}{\operatorname{det}(\boldsymbol{\Sigma})} \operatorname{adj}(\boldsymbol{\Sigma})=\frac{1}{\sigma_{1}^{2} \sigma_{2}^{2}-\sigma_{12}^{2}}\left(\begin{array}{cc}
\sigma_{2}^{2} & -\sigma_{12} \\
-\sigma_{12} & \sigma_{1}^{2}
\end{array}\right)
$$

Then, $\mathbf{Q}_{\mathbf{u}}^{\mathbf{o}}$ can be computed from Eqn. (29) as $Q_{1}^{o}=Q_{E} \frac{\sigma_{2}^{2}-\sigma_{12}}{\sigma_{1}^{2}+\sigma_{2}^{2}-2 \sigma_{12}}, Q_{2}^{o}=Q_{E} \frac{\sigma_{1}^{2}-\sigma_{12}}{\sigma_{1}^{2}+\sigma_{2}^{2}-2 \sigma_{12}}$. It can be seen that $Q_{1}^{o}, Q_{2}^{o}$ to be greater than zero requires $\frac{\sigma_{2}}{\sigma_{1}}>\rho$ and $\frac{\sigma_{1}}{\sigma_{2}}>\rho$, respectively. If satisfied, both technologies are part of the variance-minimal portfolio.
} 
For a better understanding of efficient portfolio characteristics, we next discuss sensitivity properties of the efficient fuel mix with respect to variations of the risk parameters $\sigma_{1}, \sigma_{2}, A$ and the coefficient of (fuel price) correlation $\rho:=\frac{\sigma_{12}}{\sigma_{1} \sigma_{2}}$.

Property 3.6.2. As shown in Appendix B.6, risk-cost-efficient operating hours (respectively capacities) of the considered technologies are independent from the risk-aversion parameter $A$ if (and only if) $\frac{\sigma_{1}}{\sigma_{2}}=\rho$. This parameter configuration at the same time implies that the pure cost-efficient portfolio equals the pure variance-efficient portfolio. Only if $\frac{\sigma_{1}}{\sigma_{2}}>\rho$, the efficient run time of the technology 2 increases with increasing risk aversion and vice versa. For $\frac{\sigma_{1}}{\sigma_{2}}<\rho$, which is in general satisfied as the operating costs of the peak technology are much higher than those of the base technology (see Section 4), an increasing risk aversion leads ceteris paribus to a shorter optimal run time and therewith to smaller optimal capacities of technology 2. Thus, increasing risk aversion leads in the latter case to a decline of fuel mix diversification in the considered portfolio. In summary it is

$$
\frac{\mathrm{d} t_{1}^{*}}{\mathrm{~d} A} \lesseqgtr 0, \quad \text { for } \frac{\sigma_{1}}{\sigma_{2}} \lesseqgtr \rho .
$$

Property 3.6.3. Alternatively, a comparison of optimal operating times for the pure cost-efficient portfolio, $t_{1}^{c *}$, and for the pure risk-efficient portfolio, $t_{1}^{r *}$, can provide evidence on the sensitivity. As shown in Appendix B.6, it equivalently holds

$$
\frac{\mathrm{d} t_{1}^{*}}{\mathrm{~d} A} \lesseqgtr 0, \quad \text { for } t_{1}^{c *} \gtreqless t_{1}^{r *}
$$

This is an interesting finding, since it implies by taking into account Properties 3.6.1 and 3.6.2, that the case $t_{1}^{c *}<t_{1}^{r *}$ cannot occur if both technologies are part of the variance-efficient portfolio.

For the following sensitivity properties of the solution to the two-technology problem corresponding to Eqs. (8) to (11) with $n=2$, we will hence concentrate on the more interesting case where both technologies are part of the variance-efficient portfolio, i.e. $\frac{\sigma_{1}}{\sigma_{2}}>\rho$ and $\frac{\sigma_{2}}{\sigma_{1}}>\rho$ as shown in Appendix B.6.

Property 3.6.4. For technologies 1 and 2 being part of the cost-efficient and the variance-efficient portfolio, the following parameter conditions are sufficient for the stated sensitivity properties of optimal operating hours (respectively capacities) of technology 2 :

$$
\begin{array}{ll}
\frac{\mathrm{d} t_{1}^{*}}{\mathrm{~d} \sigma_{1}} \geq 0 & \text { for all } \sigma_{1}, \sigma_{2} \geq 0,0 \leq \rho \leq 1, \\
\frac{\mathrm{d} t_{1}^{*}}{\mathrm{~d} \sigma_{2}} \leq 0 & \text { for all } \sigma_{1}, \sigma_{2} \geq 0,0 \leq \rho \leq 1, \\
\frac{\mathrm{d} t_{1}^{*}}{\mathrm{~d} \rho} \leq 0 & \text { for all } \sigma_{1}, \sigma_{2}, \geq 0,-1 \leq \rho \leq 1 .
\end{array}
$$

Reciprocal sensitivity properties are obtained for optimal operating hours (respectively capacities) of technology 1. 
Table 1: German power generation fuel mix 2007 (BMWi, 2008, source:).

\begin{tabular}{lrr}
\hline & Gross production (TWh) & Gross capacities (GW) \\
\hline Hard coal & $142.0(22.3 \%)$ & $29.3(21.3 \%)$ \\
Lignite & $155.1(24.3 \%)$ & $22.5(16.4 \%)$ \\
Nuclear & $140.5(22.0 \%)$ & $21.3(15.5 \%)$ \\
Gas & $75.9(11.9 \%)$ & $21.3(15.5 \%)$ \\
Oil & $9.7(1.5 \%)$ & $5.4(4.0 \%)$ \\
Wind & $39.7(6.2 \%)$ & $22.2(16.1 \%)$ \\
Water & $28.1(4.4 \%)$ & $10.1(7.4 \%)$ \\
Miscellaneous & $46.4(7.3 \%)$ & $5.3(3.9 \%)$ \\
\hline Total & $637.6(100 \%)$ & $137.5(100 \%)$ \\
\hline
\end{tabular}

\section{Application: Long-term efficient fuel mix of the German electricity market}

In the following section, we will deepen the insights gained from the analytics on risk-efficient generation portfolios by a real case application. For two reasons, we decided to focus for this on the German electricity market: For the first, there is a considerable need for new investments in generation assets in Germany in the next decades induced by the age of the existing plant fleet, the so far planned stepwise phaseout of nuclear generation until 2022 (Deutscher Bundestag, 2002) and the increased consciousness to act against climate change which has led to a diminishing acceptance of coal plants - so far the dominating generation technology in Germany.

The second reason for analyzing the German fuel mix is that to our best knowledge, a MVP analysis of the German electricity market has not yet been published before, so that this market will provide a worthwhile example.

\subsection{Parameter estimates}

\subsubsection{Estimation of plant costs and fuel prices}

As reported by the German Ministry for Economics and Technology in BMWi (2008), the fuel mix for power generation in Germany is dominated by coal and nuclear plants, which in 2007 added to almost $70 \%$ of the gross power production (Table 1). Gas plants serve as the predominant peak technology, supplemented by pumped store and (reserve) oil plants. For replacements and extensions of the existing plant fleet, (hard) coal and natural gas plants as well as renewable technologies represent currently the most considered options. Due to economic incentives from subsidies, renewable technologies do already contribute to more than $10 \%$ to the German electricity production.

A comparison of technical and economic key parameters for typical representatives of the main generation technologies based on 2007 values derived from Konstantin (2009) is summarized in Table 2. Total investment 
Table 2: 2007 based key parameters for typical coal, gas and nuclear plants (source: Konstantin, 2009, own analysis).

\begin{tabular}{|c|c|c|c|c|c|c|}
\hline Parameter & Unit & Hard coal & Lignite & CCGT & GT & Nuclear \\
\hline Thermal efficiency & $\mathrm{MWh}_{e} / \mathrm{MWh}_{t}$ & 0.46 & 0.43 & 0.56 & 0.34 & 0.37 \\
\hline Carbon emission rate & $\mathrm{tCO}_{2} / \mathrm{MWh}_{t}$ & 0.34 & 0.41 & 0.20 & 0.20 & 0.0 \\
\hline Total net investment costs & $€ / \mathrm{KW}$ & 1419 & 1934 & 608 & 456 & 3225 \\
\hline Technical lifetime & a & 45 & 45 & 30 & 25 & 50 \\
\hline Fixed O\&M, overhead & $€ / \mathrm{KW}$ a & 36.06 & 43.26 & 13.97 & 9.69 & 74.06 \\
\hline Variable O\&M, transport & $€ / \mathrm{KWh}_{e}$ & 2.9 & 1.7 & 5.5 & 20.0 & 0.0 \\
\hline
\end{tabular}

$\operatorname{costs}^{21}$ include engineering, procurement, construction, decommissioning and for the EPR nuclear waste management costs as well as interest payments during construction. Annualized specific investment costs per KW are quoted with respect to the gross installed capacity including plant consumption of auxiliaries. For all technologies, capital costs are calculated based on the annuity method with a uniform interest rate of $10 \%$ after tax and the quoted economic lifetimes. For consistency with the other plant key parameters, we use 2007 average fuel prices as depicted in Table 2.

To account for fuel price risks, total operating costs $c_{o p, u}$ are modeled as normal random variables calculated as the sum of the respective fuel prices plus the emission factor weighted price of $\mathrm{CO}_{2}$ emission rights divided by the technology specific efficiency rate, i.e. $c_{o p, u}=\frac{p_{f, u}+e_{u} p_{c o_{2}}}{\eta_{u}}$.

Since valuing the influence of fuel price fluctuations on the long-term investment optimum in a MVP approach requires a reliable long-term estimate of the covariance matrix which captures all underlying price risks, we estimate variance and covariance for (pairs of) total fuel prices including $\mathrm{CO}_{2}$ for the considered generation technologies over the sample period 1986-2008 as shown in Table 3. Quarterly coal import prices 1986-2008 and monthly natural gas import prices 1991-2008 are based on reports of the Federal Office of Economics and Export Control (BAFA, 2009). Annually averaged gas import prices before 1991 are provided by the German Council of Economic Experts (Sachverständigenrat, 2008). Coal and gas fuel prices reflect the average cross-border price converted to $€ / \mathrm{MWh}_{t}$ for all contracted deliveries in the respective month. Lignite price series are derived from observations by Konstantin (2009) and energy price trends reported by the German Federal Statistical Office StaBu (2009). From 2005 onwards $^{22}$, total fuel prices are computed including the costs of $\mathrm{CO}_{2}$ emission allowances (EUA) based on front year price data from ECX (2009). EUAs are modeled to be purchased at market conditions (full auctioning) as it has been announced by the EU for ETS Phase III starting in 2013. The price statistics for nuclear fuels represent total fuel cycle costs. They are composed of monthly spot prices for delivery within a year for uranium oxide $\mathrm{U}_{3} \mathrm{O}_{8}$, for the conversion in tho uranium hexafluoride $\mathrm{UF}_{6}$ and for the enrichment process. Monthly data is provided

\footnotetext{
${ }^{21}$ In economic literature sometimes also denoted as "overnight" costs.

${ }^{22}$ Phase I of the European Union Emission Trading System (EU ETS) started in 2005.
} 
Table 3: Distribution parameters for fuel costs 1986-2008 (Germany), EUA costs included from 2005 on (source: BAFA, 2009 ; UxC, 2009; ECX, 2009; StaBu, 2009, own analysis).

\begin{tabular}{lcccc||rr}
\hline & \multicolumn{3}{c}{ Coefficient of correlation } & \multicolumn{2}{c}{ Std.-dev. } & Mean 2006-08 \\
& Gas & Hard coal & Lignite & Nuclear & $€ / \mathrm{MWh}_{t}$ & $€^{-\mathrm{MWh}_{t}}$ \\
\hline Gas & 1.00 & 0.92 & 0.88 & 0.77 & 7.48 & 28.73 \\
Hard coal & 0.92 & 1.00 & 0.94 & 0.67 & 4.32 & 15.83 \\
Lignite & 0.88 & 0.94 & 1.00 & 0.62 & 3.33 & 11.41 \\
Nuclear EPR & 0.77 & 0.67 & 0.62 & 1.00 & 0.30 & $2.66^{a}$ \\
\hline
\end{tabular}

${ }^{a}$ Fuel price EPR represents total fuel cycle costs, thereof $1.55 € / \mathrm{MWh}_{t}$ for fuel supply (uranium production, conversion, enrichment and fabrication) and $1.11 € / \mathrm{MWh}_{t}$ for disposal.

by UxC (2009) for the period 1987-2008. Where missing, conversion and enrichment prices are estimated based on calculated averages. Cost of fabrication is estimated as a constant price component of $275 \$ / \mathrm{kg}$ $\mathrm{UO}_{2}$, the cost of disposal with $2500 \$ / \mathrm{kg} \mathrm{UO}$.

To avoid disruptions from short-term market imbalances, we use 2006-2008 average prices for the expectation of generation costs as depicted in Table 3 .

Under the assumption of normal distributed fuel price levels, it can be seen that the probability for reversals in the merit order, $\mathbf{P}\left(\tilde{c}_{o p, 1}>\tilde{c}_{o p, 2}\right)$, is negligibly small. Applying the transformation $\tilde{z}=\tilde{c}_{o p, 1}-$ $\tilde{c}_{o p, 2}$, where $\tilde{z} \sim \mathcal{N}\left(\bar{c}_{o p, 1}-\bar{c}_{o p, 2}, \sigma_{1}^{2}+\sigma_{2}^{2}-2 \sigma_{12}\right)^{23}$, the year-to-year likelihood for a reversal in the merit order is given by

$$
\mathbf{P}\left(\tilde{c}_{o p, 1}>\tilde{c}_{o p, 2}\right)=\mathbf{P}_{z}(\tilde{z}>0)=1-\int_{-\infty}^{0} \phi_{z}(z) \mathrm{d} z=1-\Phi_{z}(0) .
$$

where $\Phi_{z}$ denotes the cumulated distribution function of $\tilde{z}$. With the empirical data from Table 3 we obtain for the pairwise reversal likelihoods $\mathbf{P}\left(\tilde{c}_{o p, \text { coal }} \geq \tilde{c}_{\text {op,gas }}\right)=0.06 \%, \mathbf{P}\left(\tilde{c}_{o p, \text { lignite }} \geq \tilde{c}_{o p, \text { coal }}\right)=0.47 \%$, $\mathbf{P}\left(\tilde{c}_{\text {op,nuclear }} \geq \tilde{c}_{\text {op,lignite }}\right)=0.13 \%$. Therefore, reversals in the merit order are neglected in the following.

\subsubsection{Load duration curve}

We use historical load data for Germany provided in an hourly resolution by UCTE (2009) for the years 2006-2008. For comparability reasons, we adjust the data sets for the general increase in energy consumption by $1.02 \%$ in 2007 and $0.4 \%$ in 2008, respectively. A historical reference load duration curve can then be generated from the hourly means of the historic data. To accomplish the further analysis in Matlab with a

\footnotetext{
${ }^{23}$ It is well-known that the sum of $n$ jointly normal distributed random variables $X_{i}$, with $X_{i} \sim \mathcal{N}\left(\mu_{i}, \sigma_{i}^{2}\right)$ is also normal distributed with mean and variance

$$
\mu=\sum_{i=1}^{n} \mu_{i}, \quad \sigma^{2}=\sum_{i=1}^{n} \sum_{j=1}^{n} \sigma_{i j}=\sum_{i=1}^{n} \sum_{j=1}^{n} \sigma_{i} \sigma_{j} \rho, \quad \text { with } \rho=\frac{\sigma_{i j}}{\sigma_{i} \sigma_{j}} .
$$
}

For a proof see e.g. Elishakoff (1999). 
Table 4: Specification of fitted load duration functions, comparison with historical data (historical data: UCTE, 2009).

\begin{tabular}{lrrrrr}
\hline & Mean 2006-2008 & Polynomial I & Polynomial II & Linear I & Linear II \\
& & $q=5$ & $q=5$ & $q=1$ & $q=1$ \\
\hline$A_{0}$ & - & 78486 & 74746 & 78486 & 73310 \\
$A_{1}$ & - & -23.962 & -9.492 & -4.704 & -3.818 \\
$A_{2}$ & - & $2.223 \cdot 10^{-2}$ & $4.586 \cdot 10^{-3}$ & - & - \\
$A_{3}$ & - & $-1.123 \cdot 10^{-5}$ & $-1.395 \cdot 10^{-6}$ & - & - \\
$A_{4}$ & - & $3.019 \cdot 10^{-9}$ & $1.801 \cdot 10^{-10}$ & - & - \\
$A_{5}$ & - & $-4.494 \cdot 10^{-13}$ & $-8.32 \cdot 10^{-15}$ & - & - \\
$A_{6}$ & - & $3.493 \cdot 10^{-17}$ & & - & - \\
$A_{7}$ & - & $-1.106 \cdot 10^{-21}$ & & - & - \\
\hline$S S R$ & - & $3.90 \cdot 10^{9}$ & $0.49 \cdot 10^{9}$ & $62.25 \cdot 10^{9}$ & $3.58 \cdot 10^{9}$ \\
$R^{2}$ & - & 0.9979 & 0.9997 & 0.9978 & 0.9978 \\
\hline Sample calculation results for two-technology case $\left(\right.$ coal, CCGT) with $A=10^{-10}$ & \\
$t_{1}^{*}$ & 4929.1 & 5239.6 & 4955.1 & 5171.1 & 5171.0 \\
$K_{2}^{* a}$ & $31.8 \%$ & $32.1 \%$ & $28.6 \%$ & $30.9 \%$ & $26.9 \%$ \\
$K_{1}^{* a}$ & $68.2 \%$ & $67.9 \%$ & $71.4 \%$ & $69.1 \%$ & $63.1 \%$ \\
\hline
\end{tabular}

${ }^{a}$ Optimal installed capacity as a share of annual maximum load.

continnous function $\tilde{D}(t)$, we use linear and non-linear OLS regression to fit a polynomial function of the form

$$
\tilde{D}(t) \approx \begin{cases}\sum_{j=0}^{q} A_{j} \cdot t^{j}, & \text { for } t \leq 8760 \\ 0, & \text { for } t>8760 .\end{cases}
$$

Table 4 shows the coefficient estimates for polynomial functions of the degree $q=\{1,5\}$. For the first polynomial and the first linear function parameter $A_{0}$ is forced to equal the intercept of the empirical load at its maximum of $78486 \mathrm{MW}$. For the other polynomials, this parameter is also obtained from the OLS regression. Due to the large population, all parameter estimates are highly significant with t-values beyond the $0.1 \%$ level. Table 4 depicts for each function a comparison of the OLS-fitted function to the empirical data. The sum of squared residuals (SSR) is clearly the lowest for the polynomial II and would in absolute terms further improve for polynomials of higher degrees, however with decreasing marginal improvement. Without adjusted intercept, polynomial I would slighly perform better. Due to the fact that the empiric load duration curve is linear for a wide range of hours corresponding to cycling load times (see Figure 2), the OLS-estimated linear form II does also perform relatively well. However, the non-linear load characterisitcs in about the 200 hours with the highest load have an immense impact on optimal capacities of the peak-load plant technology. ${ }^{24}$

\footnotetext{
${ }^{24}$ Since only in the risk-free case with $A=0$ optimal operating hours are independent from the shape of the load duration
} 


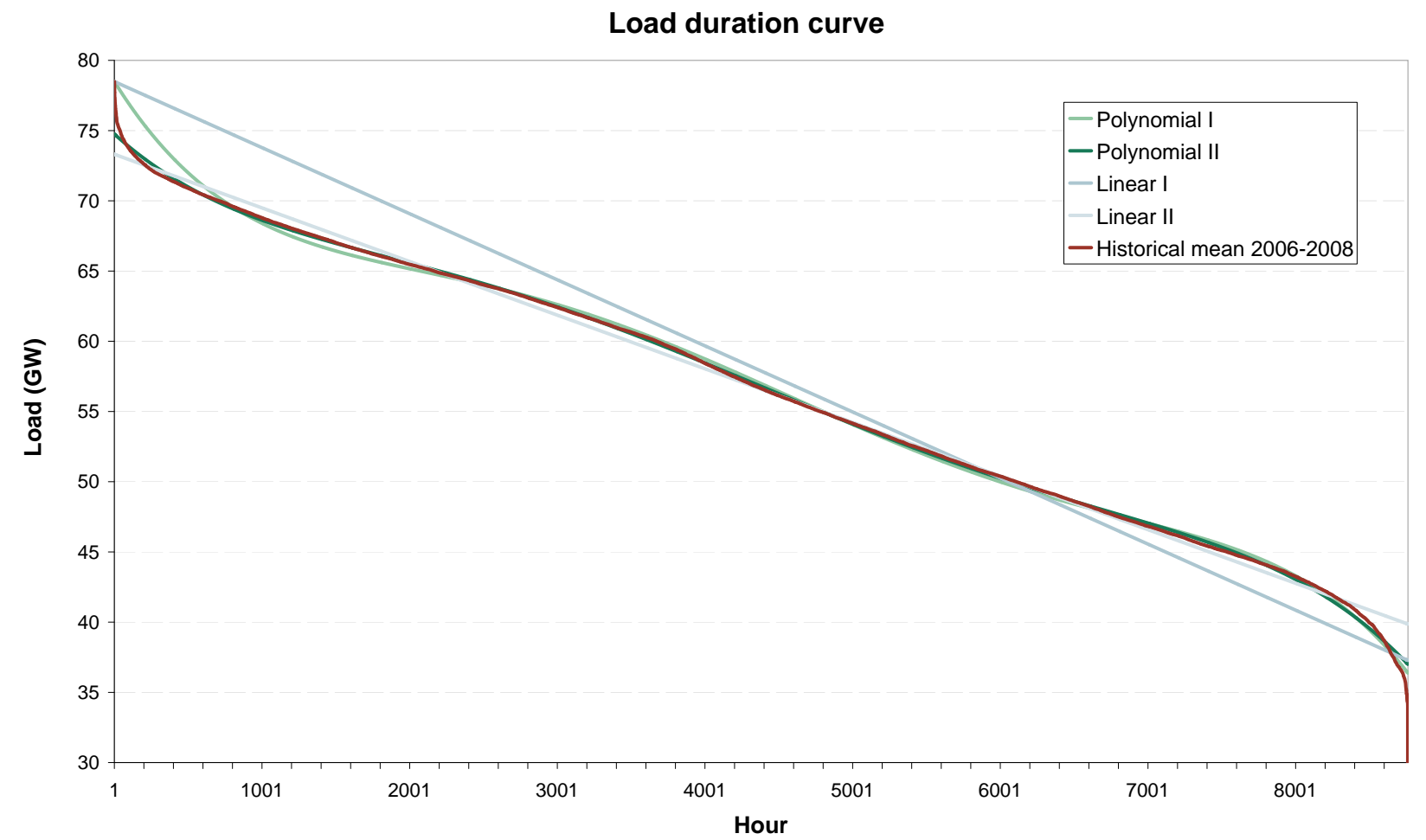

Figure 2: Historical load duration curve (red) and OLS fitted polynomial functions with degree $n=1$ (light blue), $n=1$ with adjusted intercept (dark blue), $n=5$ (dark green), and $n=5$ with adjusted intercept (green) (source: UCTE (2009); own analysis). 
The comparison of results for an exemplary calculation $\left(A=10^{-10}\right)$ is provided in the lower part of Table 4: Manually adjusting the intercept of the polynomial to the maximum load observed leads here to results that are closer to those from using the empirical load date compared to OLS-estimated intercepts. The comparison of 'Linear I' and 'Polynomial I' shows that an improved exactness in the calculation of optimal capacities goes with an increasing error in the calculation of optimal operating hours. In the following, we will use 'Polynomial I' which provides an adequate fit for capacity-based calculations.

\subsection{Results: Efficient fuel mix characteristics for the German electricity market}

We characterize the cost risk efficient generation fuel mix in a "green-field" analysis, i.e. regardless to the existing fuel mix, limited availabilities and other possible constraints. This intentionally over-idealizing study allows to first identify long-term preferable technologies before looking in more detail to a narrowed-down set of more realistic investment options in the mid-term.

With respect to fuel price risks, we investigate gas turbine (GT), combines cycle gas turbine (CCGT), lignite, coal and nuclear (EPR) plants as risky technologies. In addition, an enforced share of base-load serving technologies are incorporated as indicator for existing renewable technologies (mainly wind, water) which can be considered as nearly risk-free in terms of operating costs. ${ }^{25}$ GTs are in all cases the technology with the highest, nuclear with the lowest operating costs.

The efficient fuel mix for varying risk aversion in the first scenario with nuclear technologies is shown in Figure 3: Based on the historically estimated covariance characteristics of total fuels prices including EUAs, higher risk aversion leads always to an increase of nuclear generation in efficient portfolios. In contrast, lignite dominates the efficient portfolio in the second scenario calculation without nuclear technologies with increasing risk aversion (Figure 4). ${ }^{26}$ Vice versa, gas-fired generation decreases in all cases with increasing risk aversion. Surprisingly, hard coal does not represent a substantial share of generation in any considered case as it cannot compete with the low operating costs and low variance characteristics of nuclear and lignite generation for base load generation and with the low investment costs for gas technologies tailored to mid and peak load generation.

Clearly, this comparison shows nuclear and lignite as the most favorable generation technologies in terms of minimal costs and its corresponding variance. Although geologically feasible, the mid-term potential for

\footnotetext{
curve, they are influenced, too.

${ }^{25}$ We are aware that a solid valuation of renewable technologies in electricity generation systems requires a much deeper reflexion of technology characteristics such. Since this empirical application aims to demonstrate in a traceable manner the analytical results discussed before, we kindly refer readers with special interest in renewable technologies to the various existing publications with special focus on this topic.

${ }^{26}$ Lignite operating costs are very stable compared to other commodities even with taking into account volatile EUA markets. The reason for that is the lack of a liquid world market for lignite due to very high transportation costs. Instead, lignite power producers have usually closed long-term contracts with nearby opencast mines.
} 
lignite generation capacity extensions in Germany is realistically very limited as the exploitation of new open-cast mines faces long lasting approval processes with usually little public and political acceptance. Moreover, nuclear capacity extensions in Germany are currently prohibited by the Nuclear Exit Law which was enacted in 2002 (Deutscher Bundestag, 2002). Even though the political opinion is about to change and the time limit for the shut down of nuclear power stations may be postponed, new nuclear plant seem fairly unrealistic in the mid-term. Therefore, we will focus in the following paragraphs on the comparison of hard coal and CCGT technologies in optimal portfolios in more detail.

\subsection{Trade-off between coal and gas fired technologies}

To analyze the trade-off between the two technologies, we now consider that only CCGT and hard coal technologies are available to serve the load approximated by the function specified as 'Linear I' in Table 4. Optimal peak plant capacities, $K_{C C G T}^{*}$ (in MW) and corresponding generation costs for varying risk aversion coefficient $A$ and fuel price correlation $\rho$ are shown in Figure 5. In addition to the technology cost characteristics and the form of the load duration curve, the optimal portfolio selection is directly determined by the society's risk attitude, $A$. Since risk proclivity can be considered as abnormal for power plant investments, we will concentrate on the case $A \geq 0$.

Consistent with the results of section 3.6, we observe that with increasing risk aversion the optimal combination of capacities in the portfolio moves in general away from the risk-free optimum with $A=0$. As already discussed in Property 3.6.2, the portfolio selection is equal to the risk-free case if $\frac{\sigma_{1}}{\sigma_{2}}=\rho$. Hence at a correlation coefficient of 0.6 the variance-minimal portfolio corresponds to the cost-minimal portfolio. Consequently at this particular level of correlation, the portfolio composition is independent of the risk aversion. At higher correlations, as indicated in section 3.6, higher risk aversion reduces the share of the gas peak technology, since the diversification effect is lower than the addition in variance due to the higher price volatility of gas. If correlations were however below 0.6 , risk aversion would induce an increase in the proportion of the gas technology. These results clearly emphasize the need for appropriate correlation estimates. Given that the portfolio components have lifetimes of 30 years and more, long term correlations as those used here, estimated based on price levels, are certainly more adequate than (typically lower) short term correlations of price changes. With increasing correlation between total fuel prices of the two technologies, the optimal selection becomes additionally more and more a binary decision as depicted in Figure (7). Total expected generation costs increase as expected with increasing risk aversion as shown in Figure 6. However, the effect of increasing risk aversion is diminishing. When an increase in $A$ leads to a complete elimination of the more risky asset in the portfolio, a further increase of $A$ does not lead to a different portfolio selection. 


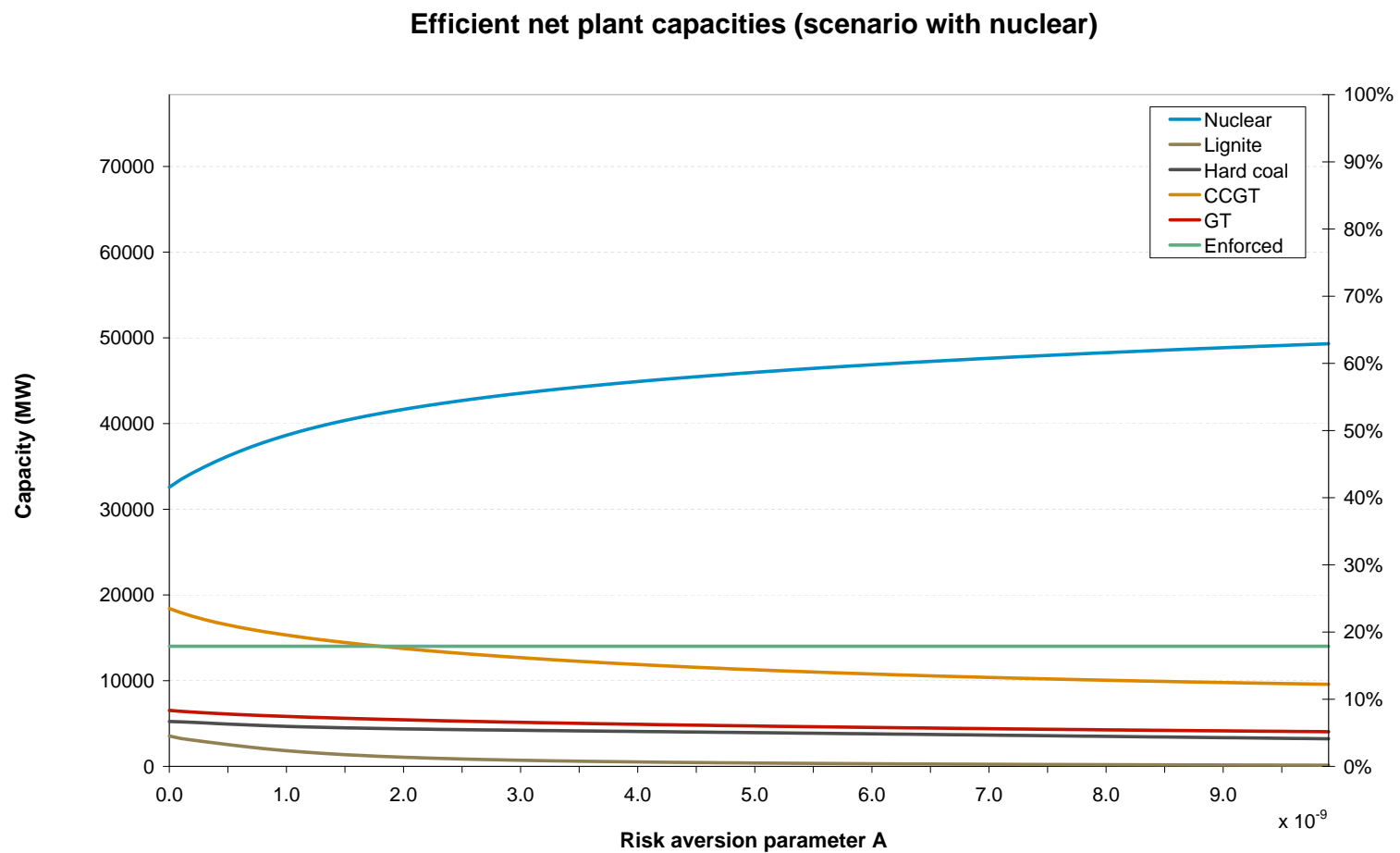

Figure 3: Efficient fuel mix of GT, CCGT, lignite, coal and nuclear technologies (in GW) for varying risk aversion coefficient $A$.

Efficient net plant capacities (scenario without nuclear)

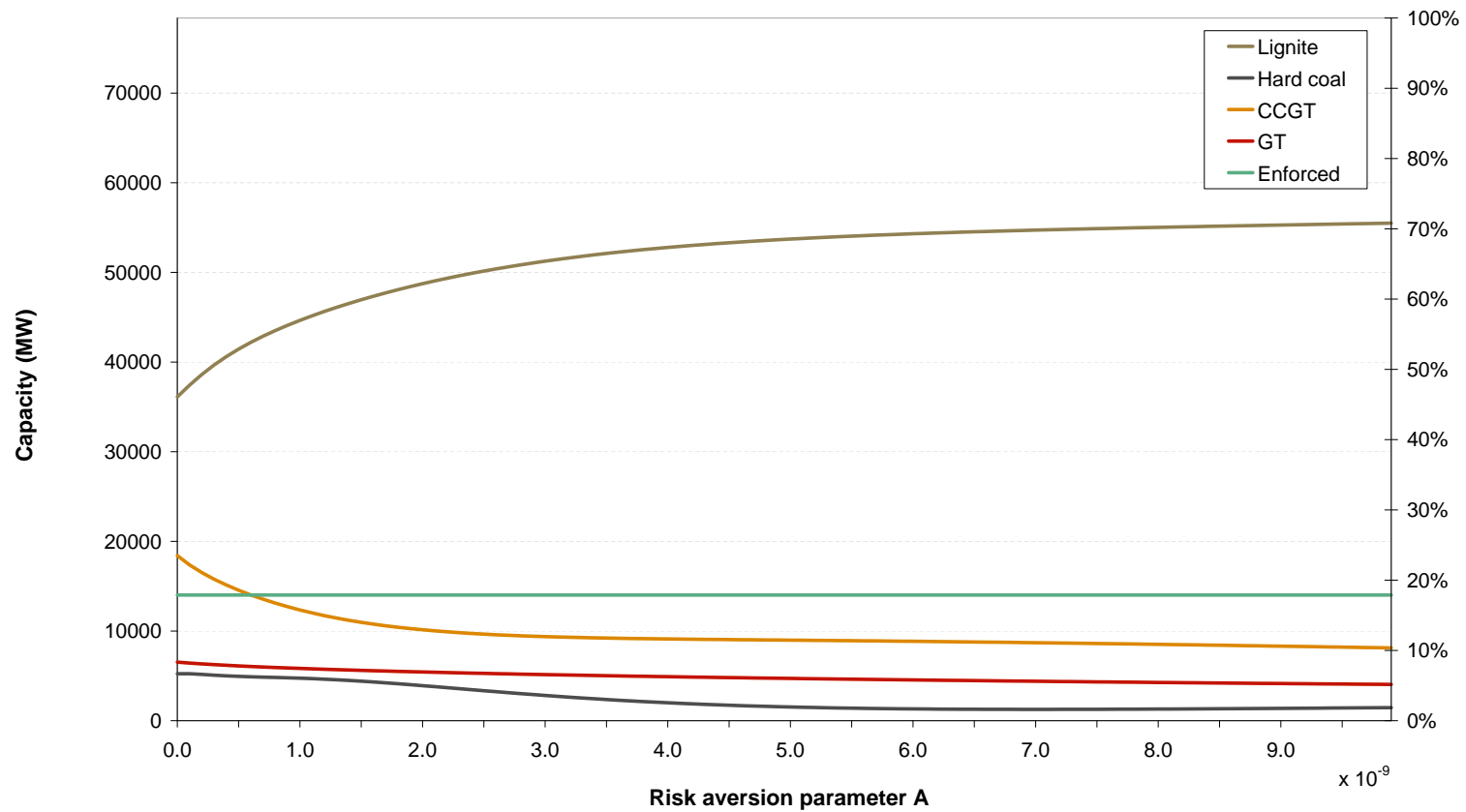

Figure 4: Efficient fuel mix of GT, CCGT, lignite, and coal technologies (in GW) for varying risk aversion coefficient $A$. 
Optimal peak capacity $\mathrm{K}_{\mathrm{CCGT}}^{*}$ for varying risk aversion and fuel price correlation

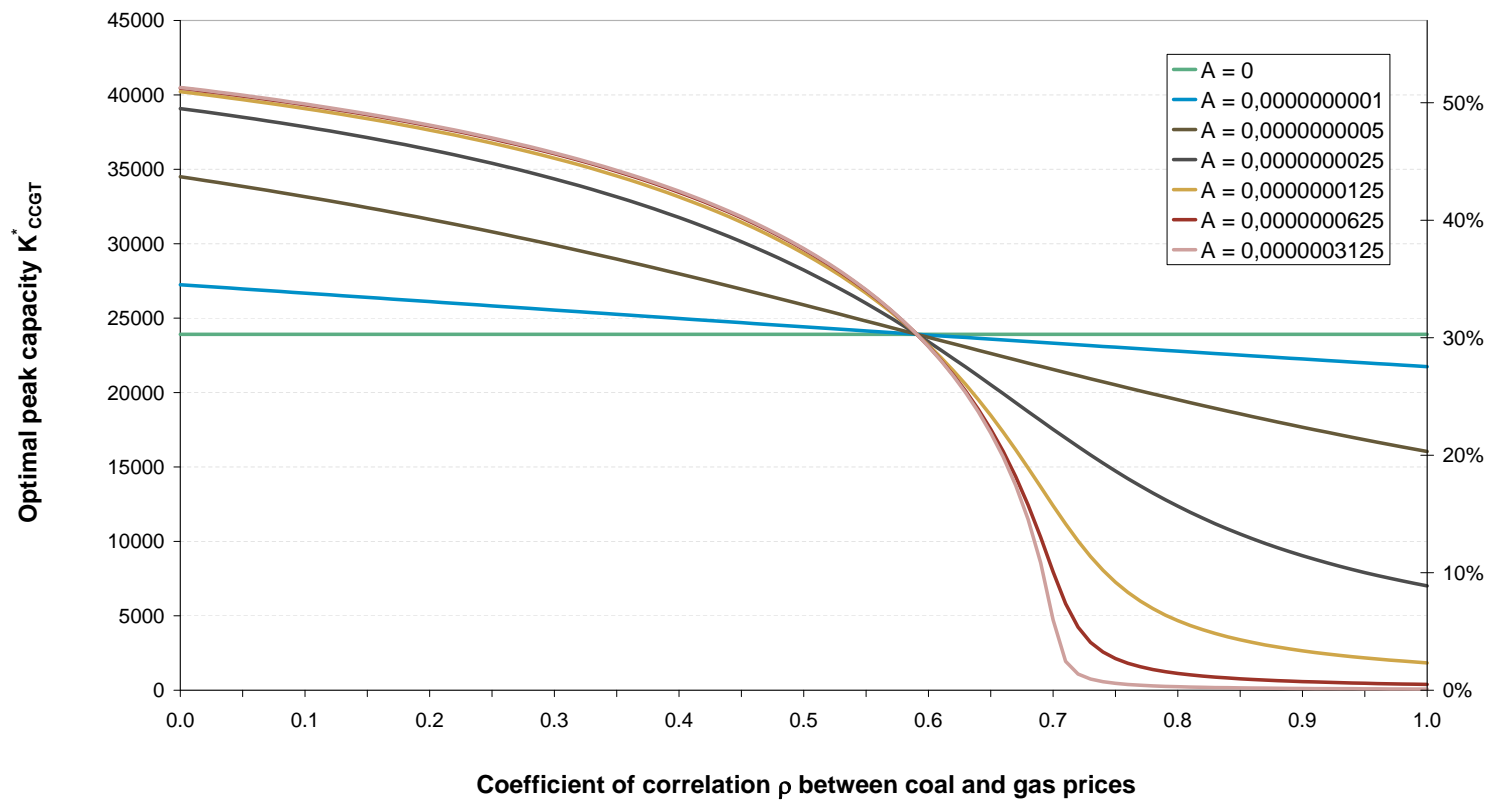

Figure 5: Optimal peak plant capacities, $K_{C C G T}^{*}$ (in MW) in two technology-case for varying risk aversion $A$ and fuel price correlation $\rho$.

Expected generation costs for varying risk aversion and fuel price correlation

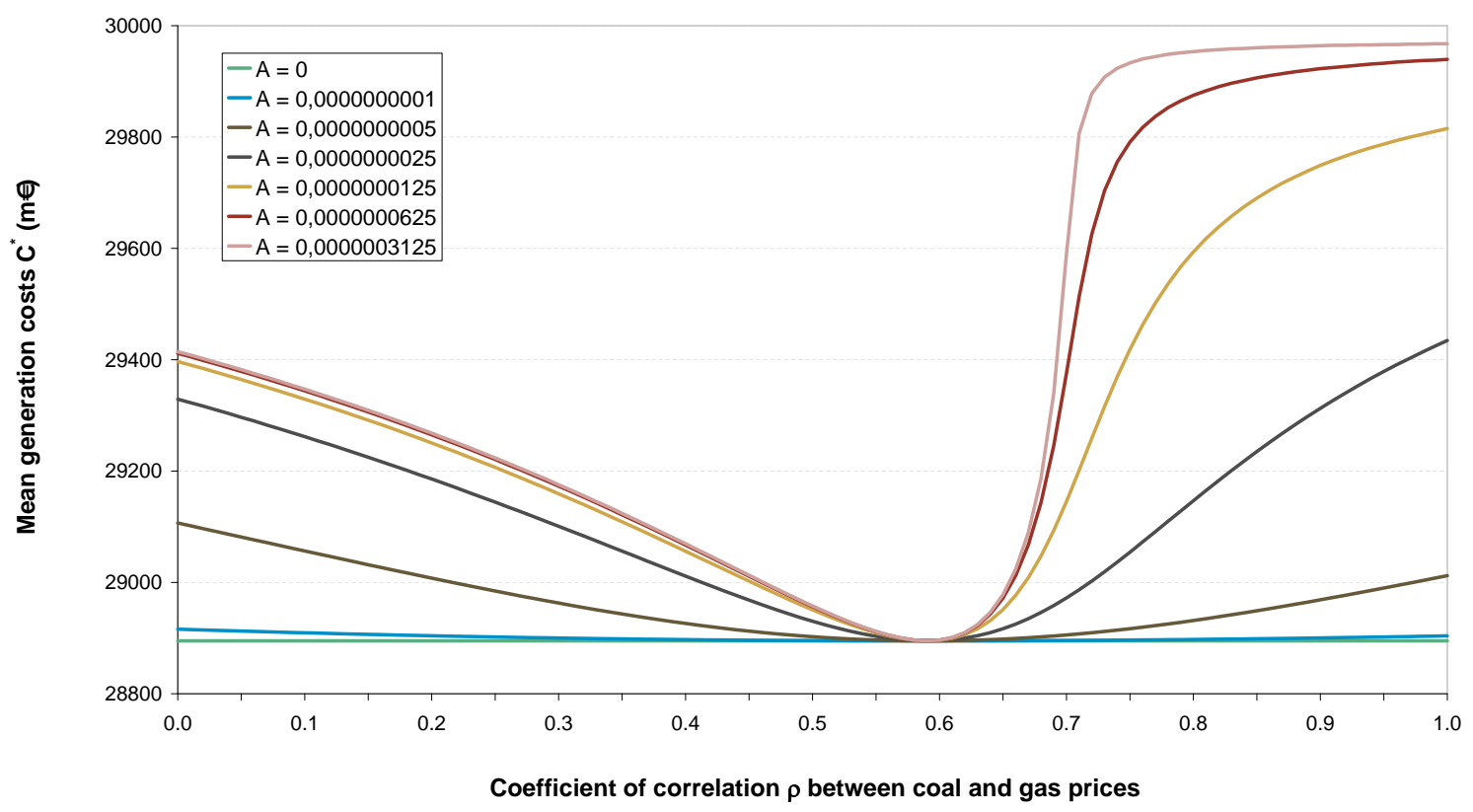

Figure 6: Expected total generation costs $C^{*}$ (in billion EUR) in two technology-case for varying risk aversion $A$ and fuel price correlation $\rho$. 
Risk adjusted optimal peak capacity $\mathrm{K}_{\mathrm{CCGT}}^{*}$ (fuel price correlation $\rho=0.5$ )

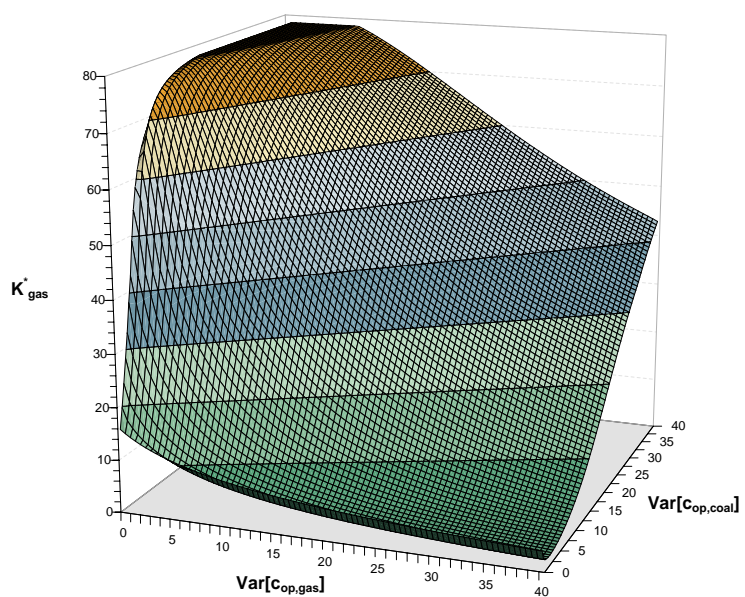

Risk adjusted optimal peak capacity $\mathrm{K}_{\mathrm{CCG}}^{\star}$ (fuel price correlation $\rho=1.0$ )

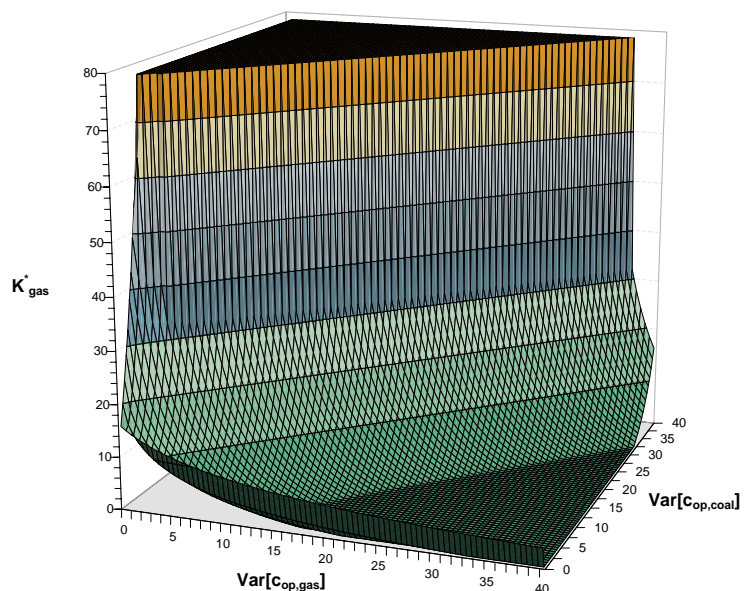

Figure 7: Optimal peak plant capacities, $K_{C C G T}^{*}$, (in GW) for $A=10^{-8}$ for varying variance of operating costs for coal, $\operatorname{Var}\left[c_{o p, c o a l}\right]$, and CCGT, $\operatorname{Var}\left[c_{o p, g a s}\right]$, at fuel price correlation of $\rho=0.5$ (left graph) and $\rho=1.0$ (right graph).

\section{Concluding remarks}

This paper has analyzed the impact of fuel mix diversification on the long-term optimum of electricity generation portfolios. By integrating Mean-Variance Portfolio theory into a classical peak-load pricing framework, an optimality condition for efficient portfolios has been derived and analyzed.

The proposed model has been used to demonstrate quantitatively the derivation of efficient generation fuel mixes for Germany. The calculations show that fuel mix diversification can considerably influence the total standard deviation of generation costs by more than $10 \%$.

With respect to the current debate on security of supply, the results indicate that increasing risk aversion implies a higher share of lignite and nuclear generation in efficient portfolios and conversely to a decrease of gas-fired generation. Independently from the risk attitude, the optimal fuel mix is highly sensitive to the price and/or the allocation method of $\mathrm{CO}_{2}$ emission rights. The results indicate that with full auctioning of $\mathrm{CO}_{2}$, efficient portfolios at historically observed $\mathrm{CO}_{2}$ price levels consist of more nuclear and lignite and less coal-fired generation compared to the current fuel mix. If nuclear and lignite capacities are reduced or fixed at the current level, hard coal is the most economical technology instead.

The exemplary results for the German generation mix demonstrate that fuel-mix diversification does not provide reduced risk characteristics per se. Blind diversification without consideration of technology costs and price risks as well as the correlation risks may even be counterproductive. 


\section{A. Symbols and model notation}

\section{Indices}

\begin{tabular}{|c|c|}
\hline & generation technology \\
\hline hours & time step during analysis period $[0 ; T]$ \\
\hline
\end{tabular}

\section{Parameters and variables}

\begin{tabular}{|c|c|c|}
\hline$A$ & $1 / €$ & Risk aversion parameter \\
\hline$D_{t}$ & MW & Total system demand at time $t$ \\
\hline$t_{u}$ & hours & Lower bound of operating hours of technology $u$ during analysis period $[0 ; T$ \\
\hline$p_{u, t}$ & $€ / \mathrm{MWh}_{t h}$ & Fuel price of technology $u$ in period $t$ \\
\hline$\eta_{u}$ & $\mathrm{MWh}_{e} / \mathrm{MWh}_{t h}$ & thermal efficiency of technology $u$ \\
\hline$h_{u}$ & $\mathrm{MWh}_{t h} / \mathrm{MWh}_{e}$ & heat rate of technology $u$ \\
\hline$e_{u}$ & $\mathrm{tCO}_{2} / \mathrm{MWh}_{t h}$ & emission rate of technology $u$ \\
\hline$K_{u}$ & MW & Installed capacity of technology $u$ \\
\hline$Q_{u}$ & MWh & Energy produced of technology $u$ in period $[0 ; T]$ \\
\hline$Q_{E}$ & MWh & Total energy produced in the system in period $[0 ; T]$ \\
\hline$y_{u, t}$ & MW & Output level of plant $u$ at time $t$ \\
\hline$C_{i n v, u}$ & $€$ & Annuity of overnight costs (total investment costs) of technology $u$ \\
\hline$c_{i n v, u}$ & $€ / \mathrm{MW}_{e}$ & Annuity of specific overnight costs of plant $u$ per installed capacity $K$ \\
\hline$C_{o p, u, t}$ & $€$ & Operating costs of plant $u$ in period $t$ \\
\hline$c_{o p, u, t}$ & $€ / \mathrm{MWh}_{e}$ & Specific operating costs of plant $u$ in period $t$ per output $y_{u, t}$ \\
\hline $\bar{c}_{o p, u}$ & $€ / \mathrm{MWh}_{e}$ & Mean operation costs of plant $u$ \\
\hline$\sigma_{u}$ & $€ / \mathrm{MWh}_{e}$ & Standard deviation of total operation costs of technology $u$ \\
\hline$\sigma_{u v}$ & $€^{2} / \mathrm{MWh}_{e}^{2}$ & Covariance of total operation costs of technologies $u$ and $v$ \\
\hline$\rho$ & - & Coefficient of correlation \\
\hline
\end{tabular}

\section{Vectors, matrices and operators}

i

$\mathbf{i}_{n}$

I

L

$\operatorname{Var}[\cdot]$

$\mathbb{E}[\cdot]$
One vector

Unit vector of dimension $n$

Identity matrix

Lag operator

Variance operator

Expected value operator 


\section{B. Mathematical Appendix}

\section{B.1. Portfolio variance and variance of operating costs}

Proof. As in Section 3.2, we assume specific operating costs per MWh to be uncertain but constant within the planning period. More specific, let specific operating costs for technology $u$ be represented by multivariate distributed random variables, $\tilde{c}_{o p, u}$, with mean $\bar{c}_{o p, u}$, variance $\operatorname{Var}\left(c_{o p, u}\right)=\sigma_{u}^{2}$ and corresponding covariance $\sigma_{u v}$. Then, the relation between variance of total operating costs for technology $u$, $\operatorname{Var}\left(C_{o p, u}\right)$, and the variance of specific operating costs, $\operatorname{Var}\left(c_{o p, u}\right)=\sigma_{u}^{2}$, can be calculated as follows:

$$
\begin{aligned}
\operatorname{Var}\left[C_{o p, u}\right] & =\operatorname{Var}\left[\int_{t} y_{u, t} c_{o p, u} \mathrm{~d} t\right]=\mathbb{E}\left[\left(\int_{t} y_{u, t} c_{o p, u}-y_{u, t} \bar{c}_{o p, u} \mathrm{~d} t\right)^{2}\right]=\mathbb{E}\left[\left(\int_{t} y_{u, t}\left(c_{o p, u}-\bar{c}_{o p, u}\right) \mathrm{d} t\right)^{2}\right] \\
& =\mathbb{E}\left[\left(\int_{t} y_{u, t} \mathrm{~d} t\right)^{2}\left(c_{o p, u}-\bar{c}_{o p, u}\right)^{2}\right]=\left(\int_{t} y_{u, t} \mathrm{~d} t\right)^{2} \mathbb{E}\left[\left(c_{o p, u}-\bar{c}_{o p, u}\right)^{2}\right] \\
& =\sigma_{u}^{2}\left(\int_{t} y_{u, t} \mathrm{~d} t\right)^{2}=\operatorname{Var}\left[Q_{u} c_{o p, u}\right]=Q_{u}^{2} \operatorname{Var}\left[c_{o p, u}\right] .
\end{aligned}
$$

The variance of specific operating costs, $\operatorname{Var}\left(c_{o p, u}\right)=\sigma_{u}^{2}$, can be calculated from the technology-specific heat rate, $h_{u}$, and from the variance of the underlying fuel price, $\operatorname{Var}\left(p_{u}\right)$, as

$$
\operatorname{Var}\left[c_{o p, t}\right]=h_{u}^{2} \operatorname{Var}\left[p_{u, t}\right]
$$

Thus, the total variance of operating costs for the set of all plants $u=\{1, \ldots, n\}$ can be calculated as:

$$
\begin{aligned}
\operatorname{Var}\left[C_{o p}\right] & =\operatorname{Var}\left[\sum_{u}\left(\int_{t} y_{u, t} c_{o p, u} \mathrm{~d} t\right)\right]=\sum_{u} \sum_{v}\left(\int_{t} y_{u, t} \mathrm{~d} t\right)\left(\int_{t} y_{v, t} \mathrm{~d} t\right) \sigma_{u v}= \\
& =\sum_{u} \sigma_{u}^{2}\left(\int_{t} y_{u, t} \mathrm{~d} t\right)^{2}+\sum_{u} \sum_{v, v \neq u} \sigma_{u v}\left(\int_{t} y_{u, t} \mathrm{~d} t\right)\left(\int_{t} y_{v, t} \mathrm{~d} t\right)=\sum_{u} \sigma_{u}^{2} Q_{u}^{2}+\sum_{u} \sum_{v, v \neq u} \sigma_{u v} Q_{u} Q_{v} .
\end{aligned}
$$

\section{B.2. Optimal technology selection for pure cost minimal portfolios with $n$ technologies}

Proposition B.2.1. Technology $u$ is part of the cost-minimal portfolio, i.e. $K_{u}^{*}>0$, only if $t_{u}^{o}<t_{u-1}^{o}$.

Proposition B.2.2. If $t_{u}^{o}<t_{u-1}^{o}$ for all $1 \leq u<n$, then the cost-minimal portfolio will consist of all technologies, i.e. $K_{u}^{*}>0$ for all $1 \leq u<n$.

A graphical interpretation of these propositions is depicted in Figure 8: Comparison of the full cost curves shows that although technology 3 (dashed line) is not "dominated" by any other technology $v$ such that $c_{o p, 2^{\prime}} t+c_{i n v, 2^{\prime}}>c_{o p, v} t+c_{i n v, v}$ for all admissible $t$, it is not part of the cost-minimal portfolio because the condition formulated in Proposition B.2.2 is violated. The cost-efficient technology mix is characterized by the lowest envelope of the different cost functions which yield piece-wise linear efficient cost curve per capacity unit as function of operating time (gray line in Figure 8). Only if all intersections of the full cost curves are obtained in a decreasing order the cost-minimal portfolio will consist of all technologies. 


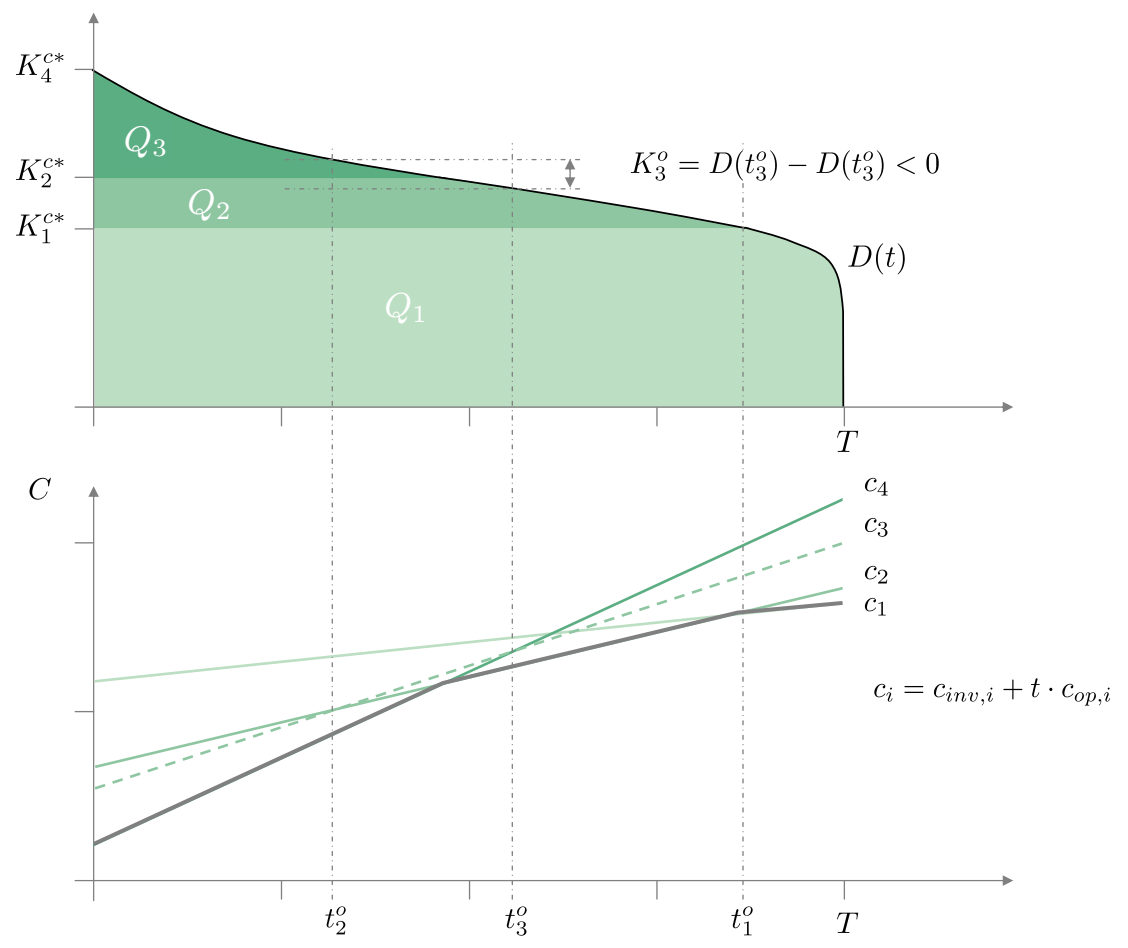

Figure 8: Graphical solution of the deterministic peak-load pricing problem from load duration curve and full-cost curves. With $t_{3}^{o}>t_{2}^{o}$, technology 3 will not be included in the cost-efficient portfolio. 
Proof of Proposition B.2.1. The proposition is proved by contradiction: From $K_{u}=D\left(t_{u}^{*}\right)-D\left(t_{u-1}^{*}\right)>0$, it can be concluded from KKT condition (10) that $\mu_{u}=0$. Assuming contrarily to the proposition that $t_{u}^{o} \geq t_{u-1}^{o}$. We can compute straightforwardly $t_{u}^{*}=t_{u}^{o}+\frac{\mu_{u+1}}{c_{o p, u+1}-c_{o p, u}}$ and $t_{u-1}^{*}=t_{u-1}^{o}-\frac{\mu_{u-1}}{c_{o p, u}-c_{o p, u-1}}$ from Eqn. (18). Given that $\mu_{u-1}, \mu_{u+1} \geq 0$, this implies $t_{u}^{*}>t_{u-1}^{*}$. The strict monotony of $D(t)$ then yields $D\left(t_{u}^{*}\right)-D\left(t_{u-1}^{*}\right)<0$, which is in contradiction with the initial hypothesis $K_{u}>0$.

Proof of Proposition B.2.2. To show the implication

$$
t_{u}^{o}<t_{u-1}^{o} \text { for all } u \Rightarrow K_{u}^{*}>0 \text { for all } u
$$

we proceed again by contradiction. Taking $t_{u}^{o}<t_{u-1}^{o}$ for all $u$ as given, we assume for one single $u$ that $K_{u}=D\left(t_{u}^{*}\right)-D\left(t_{u-1}^{*}\right)=0$. Without much limitation of the generality for the succeeding technology $u+1$ and the preceding technology $u-1$, we assume $K_{u+1}>0, K_{u-1}>0$, implying $\mu_{u+1}=0$ and $\mu_{u-1}=0 .{ }^{27}$ Straightforwardly, $t_{u}^{*}=t_{u}^{o}-\frac{\mu_{u}}{c_{o p, u+1}-c_{o p, u}}$ and $t_{u-1}^{*}=t_{u-1}^{o}+\frac{\mu_{u}}{c_{o p, u}-c_{o p, u-1}}$ may be computed which yields $t_{u}^{*}<t_{u-1}^{*}$ for all $\mu_{u} \geq 0$. This, however, implies $K_{u}=D\left(t_{u}^{*}\right)-D\left(t_{u-1}^{*}\right)>0$, in contradiction to the starting assumption.

\section{B.3. Solution to the pure variance minimization problem with $n$ technologies}

Based on Eqs. (25) and (26), the optimality conditions (27) and (28) can be derived as follows: Starting with $\lambda^{Q} \mathbf{i}=\mathbf{\Sigma} \mathbf{Q}-\boldsymbol{\mu}^{Q}$ from Eqn. (25), the positive definiteness of matrix $\boldsymbol{\Sigma}$ allows multiplication with $\mathbf{i}^{T} \boldsymbol{\Sigma}^{-1}$ from the left followed by division through the scalar $\mathbf{i}^{T} \boldsymbol{\Sigma}^{-\mathbf{1}} \mathbf{i}$ yielding

$$
\begin{aligned}
\left(\mathbf{i}^{T} \boldsymbol{\Sigma}^{-\mathbf{1}}\right) \lambda^{Q} \mathbf{i} & =\left(\mathbf{i}^{T} \boldsymbol{\Sigma}^{-\mathbf{1}}\right) \mathbf{\Sigma} \mathbf{Q}-\left(\mathbf{i}^{T} \boldsymbol{\Sigma}^{-\mathbf{1}}\right) \boldsymbol{\mu}^{Q} \\
\Leftrightarrow \quad \lambda^{Q} & =\frac{1}{\mathbf{i}^{T} \boldsymbol{\Sigma}^{-\mathbf{1}} \mathbf{i}}\left(\left(\mathbf{i}^{T} \boldsymbol{\Sigma}^{-\mathbf{1}}\right) \boldsymbol{\Sigma} \mathbf{Q}-\left(\mathbf{i}^{T} \boldsymbol{\Sigma}^{-\mathbf{1}}\right) \boldsymbol{\mu}^{Q}\right)=\frac{1}{\mathbf{i}^{T} \boldsymbol{\Sigma}^{-\mathbf{1}} \mathbf{i}}\left(\mathbf{i}^{T} \mathbf{Q}-\mathbf{i}^{T} \boldsymbol{\Sigma}^{-\mathbf{1}} \boldsymbol{\mu}^{Q}\right)
\end{aligned}
$$

Finally, we apply $Q_{E}=\mathbf{i}^{T} \mathbf{Q}$ from Eqn. (26) to obtain optimality condition (27):

$$
\lambda^{Q}=\frac{1}{\mathbf{i}^{T} \boldsymbol{\Sigma}^{-\mathbf{1}} \mathbf{i}}\left(A Q_{E}-\mathbf{i}^{T} \boldsymbol{\Sigma}^{-\mathbf{1}} \boldsymbol{\mu}^{Q}\right)
$$

By inserting $\lambda^{Q}$ in Eqn. (25), $\mathbf{Q}$ can be computed as

$$
\begin{aligned}
\mathbf{Q} & =\boldsymbol{\Sigma}^{-\mathbf{1}}\left(\lambda^{Q} \mathbf{i}+\boldsymbol{\mu}^{Q}\right)=\boldsymbol{\Sigma}^{-\mathbf{1}}\left(\frac{1}{\mathbf{i}^{T} \boldsymbol{\Sigma}^{-\mathbf{1}} \mathbf{i}}\left(Q_{E}-\mathbf{i}^{T} \boldsymbol{\Sigma}^{-\mathbf{1}} \boldsymbol{\mu}^{Q}\right) \mathbf{i}+\boldsymbol{\mu}^{Q}\right) \\
& =\boldsymbol{\Sigma}^{-\mathbf{1}}\left(\frac{1}{\mathbf{i}^{T} \boldsymbol{\Sigma}^{-\mathbf{1}} \mathbf{i}}\left(Q_{E} \mathbf{i}-\mathbf{i i}^{T} \boldsymbol{\Sigma}^{-\mathbf{1}} \boldsymbol{\mu}^{Q}\right)+\boldsymbol{\mu}^{Q}\right)=\frac{Q_{E}}{\mathbf{i}^{T} \boldsymbol{\Sigma}^{-\mathbf{1}} \mathbf{i}} \boldsymbol{\Sigma}^{-\mathbf{1}} \mathbf{i}+\left(\boldsymbol{\Sigma}^{-\mathbf{1}}-\frac{1}{\mathbf{i}^{T} \boldsymbol{\Sigma}^{-\mathbf{1}} \mathbf{i}} \boldsymbol{\Sigma}^{-\mathbf{1}} \mathbf{i i}^{T} \boldsymbol{\Sigma}^{-\mathbf{1}}\right) \boldsymbol{\mu}^{Q}
\end{aligned}
$$

\footnotetext{
${ }^{27}$ In the more general case with possibly several subsequent technologies with zero capacities, a recursive procedure of elimination of inefficient technologies has to be started.
} 
Proposition B.3.1. The pure variance-minimization problem is convex in $\mathbf{Q}$. If and only if $\boldsymbol{\Sigma}$ is positive definite, then the optimization problem is strictly convex in $\mathbf{Q}$.

Proof of Proposition B.3.1. For the pure variance minimal portfolio, the objective function from problem (8) can be rewritten as

$$
L^{r}(\mathbf{Q})=\mathbf{Q}^{T} \mathbf{\Sigma} \mathbf{Q}
$$

The Hessian of the objective function can be derived straightforwardly with matrix calculus as $\mathbf{H}^{r}=$ $\boldsymbol{\Sigma}$. Taking into account that an arbitrary covariance matrix $\boldsymbol{\Sigma}$ is positive semi-definite (cf. Horn and Johnson, 1985, p. 392), convexity of $L^{r}$ can be concluded. Furthermore, the Hessian is positive definite and consequently $L^{r}$ strictly convex if and only if $\boldsymbol{\Sigma}$ is positive definite. Using $\mathbf{Q}$ as decision variable, constraints (10) and (11) can be rewritten as $-\mathbf{Q} \leq \mathbf{0}$ and $Q_{E}-\mathbf{Q i} \leq \mathbf{0}$, so that linearity and hence also convexity of both constraints become obvious.

Proposition B.3.2. Let be $\mathbf{Q}^{o}:=\omega Q_{E} \boldsymbol{\Sigma}^{-1} \mathbf{i}$, with $\omega:=\left(\mathbf{i}^{T} \boldsymbol{\Sigma}^{-1} \mathbf{i}\right)^{-1}$ and $\mathbf{i}=(1, \ldots, 1)^{T}$. The varianceminimal portfolio consists of all available technologies, i.e. $Q_{u}>0$ for all $1 \leq u<n$, if and only if $Q_{u}^{o}>0$ for all $1 \leq u<n$. Then, $\mathbf{Q}=\mathbf{Q}^{o}$ is a solution to the variance minimization. The solution is unique if $\mathbf{\Sigma}$ is positive definite.

Proof of Proposition B.3.2. For notational brevity, we define $\mathbf{R}:=\omega \boldsymbol{\Sigma}^{-\mathbf{1}}\left(\left(\mathbf{i}^{T} \boldsymbol{\Sigma}^{-\mathbf{1}} \mathbf{i}\right) \mathbf{I}-\mathbf{i i}^{T} \boldsymbol{\Sigma}^{-\mathbf{1}}\right)$. Then, we can rewrite Eqn. (28)

$$
\mathbf{Q}=\mathbf{Q}^{\mathbf{o}}+\mathbf{R} \boldsymbol{\mu}^{Q}
$$

Remark that the symmetric matrix $\mathbf{R}$ is in general indefinite, even for $\boldsymbol{\Sigma}^{\mathbf{- 1}}$ being positive definite. ${ }^{28}$ Suppose $Q_{u}>0$, which implies $\mu_{u}^{Q}=0$ according to KKT condition (10). Consequently, $Q_{u}>0$ for all $1 \leq u<n$ implies $Q_{u}^{o}>0$ for all $1 \leq u<n$. Therewith, $Q_{u}^{o}>0$ for all $1 \leq u<n$ represents the necessary condition for the variance-minimal portfolio to consist of all available technologies.

The condition is even sufficient for the variance-minimal solution, since $Q_{u}=Q_{u}^{o}, \mu_{u}=0$ for all $1 \leq u<n$ represents a solution to the equation system (27)-(28) and therewith a local variance minimum to the considered portfolio problem. Taking into account convexity of the optimization problem as shown in Proposition B.3.1, it is clear that any local variance minimum is also global. For $\boldsymbol{\Sigma}$ being positive definite, the optimization problem is strictly convex and hence the obtained solution is unique.

\footnotetext{
${ }^{28}$ Only nonnegative linear combinations of positive semi-definite matrices are again positive definite (cf. Horn and Johnson, 1985, Observation 7.1.3), however, $\mathbf{R}$ represents a negative linear combination of definite matrices.
} 


\section{B.4. Solution to the general portfolio problem with $n$ technologies}

The central solution condition (cf. Eqn. (34)) for the general risk-adjusted portfolio problem can be derived from $\frac{\partial \mathcal{L}_{n}}{\partial \mathbf{K}^{\mathrm{c}}}$ as follows:

$$
\begin{array}{rrr} 
& \left.A \operatorname{diag}\left((\mathbf{I}-\mathbf{L}) t\left(\mathbf{K}^{\mathbf{c}}\right)\right)\right) \boldsymbol{\Sigma}(\mathbf{L}-\mathbf{I}) Q^{I}\left(\mathbf{K}^{\mathbf{c}}\right)=(\mathbf{I}-\mathbf{L})^{T}\left(\mathbf{c}_{\mathbf{i n v}}+\boldsymbol{\mu}\right)+\operatorname{diag}\left(t\left(\mathbf{K}^{\mathbf{c}}\right)\right)(\mathbf{I}-\mathbf{L})^{T} \mathbf{c}_{\mathbf{o p}} \\
\Leftrightarrow & A \boldsymbol{\Sigma}(\mathbf{L}-\mathbf{I}) Q^{I}\left(\mathbf{K}^{\mathbf{c}}\right)=\operatorname{diag}\left((\mathbf{I}-\mathbf{L}) t\left(\mathbf{K}^{\mathbf{c}}\right)\right)^{-1}\left((\mathbf{I}-\mathbf{L})^{T}\left(\mathbf{c}_{\mathbf{i n v}}+\boldsymbol{\mu}\right)+\operatorname{diag}\left(t\left(\mathbf{K}^{\mathbf{c}}\right)\right)(\mathbf{I}-\mathbf{L})^{T} \mathbf{c}_{\mathbf{o p}}\right) \\
\Leftrightarrow & -A \boldsymbol{\Sigma} Q\left(\mathbf{K}^{\mathbf{c}}, \mathbf{L} \mathbf{K}^{\mathbf{c}}\right)=\operatorname{diag}\left((\mathbf{I}-\mathbf{L}) t\left(\mathbf{K}^{\mathbf{c}}\right)\right)^{-1}\left((\mathbf{I}-\mathbf{L})^{T}\left(\mathbf{c}_{\mathbf{i n v}}+\boldsymbol{\mu}\right)+\operatorname{diag}\left(t\left(\mathbf{K}^{\mathbf{c}}\right)\right)(\mathbf{I}-\mathbf{L})^{T} \mathbf{c}_{\mathbf{o p}}\right) \\
\Leftrightarrow & \quad \mathbf{Q}=-\frac{1}{A} \boldsymbol{\Sigma}^{-\mathbf{1}} \operatorname{diag}\left((\mathbf{I}-\mathbf{L}) t\left(\mathbf{K}^{\mathbf{c}}\right)\right)^{-1}\left((\mathbf{I}-\mathbf{L})^{T}\left(\mathbf{c}_{\mathbf{i n v}}+\boldsymbol{\mu}\right)+\operatorname{diag}\left(t\left(\mathbf{K}^{\mathbf{c}}\right)\right)(\mathbf{I}-\mathbf{L})^{T} \mathbf{c}_{\mathbf{o p}}\right)
\end{array}
$$

Proposition B.4.1. Let be $A>0$ and $c_{i n v, u}>c_{i n v, u+1}$ for all $(u=1, \ldots, n-1)$. Then the combined portfolio problem (8)-(11) is convex in $\mathbf{Q}$. If and only if $\boldsymbol{\Sigma}$ is positive definite, then the optimization problem is strictly convex in $\mathbf{Q}$.

Proof of Proposition B.4.1. As shown in Proposition B.3.1, the pure variance-minimization problem is convex (strictly convex) in $\mathbf{Q}$ if and only if $\boldsymbol{\Sigma}$ is positive semi-definite (positive definite). For the second part of the proof, we consider the objective function of the pure cost-minimization problem

$$
L^{c}(\mathbf{Q})=\mathbf{c}_{\mathbf{i n v}}^{T}(\mathbf{I}-\mathbf{L}) \mathbf{K}^{\mathbf{c}}(\mathbf{Q})+\overline{\mathbf{c}}_{\mathbf{o p}}^{T} \mathbf{Q}
$$

Note that the first summand is linear in $\mathbf{K}^{\mathbf{c}}(\mathbf{Q})$, which itself is a nonlinear function of $\mathbf{Q}$. From the definition of $Q_{u}^{I}$ in Eqn. (7) it is known that $Q_{u}^{I}\left(K_{u}^{c}\right)=f\left(K_{u}^{c}\right)$ is an increasing and concave function of $K_{u}^{c}$ since $\frac{\mathrm{d}\left(Q_{u}^{I}\left(K_{u}^{c}\right)\right)^{2}}{\mathrm{~d}^{2} K_{u}^{c}}=\frac{\mathrm{d} R\left(K_{u}^{c}\right)}{\mathrm{d} K_{u}^{c}} \leq 0$ since $D(t)$ is monotone decreasing. Hence, it can be concluded that the inverse function $K_{u}^{c}\left(Q_{u}^{I}\right)=f^{-1}\left(Q_{u}^{I}\right)$ is convex in $Q_{u}^{I}$. In fact, $Q_{u}^{I}$ can be expressed as the nonnegative linear combination $Q_{u}^{I}=\sum_{i=1}^{u} Q_{i}$, hence $K_{u}^{c}\left(Q_{u}^{I}\right)=K_{u}^{c}\left(Q_{1}, \ldots, Q_{u}\right)$ is also convex in each $Q_{i},(i=1, \ldots, u)$. Finally, $\mathbf{c}_{\mathbf{i n v}}{ }^{T}(\mathbf{I}-\mathbf{L}) \mathbf{K}^{\mathbf{c}}(\mathbf{Q})$ is convex as a nonnegative linear combination of convex functions if $c_{i n v, u}>$ $c_{i n v, u+1}$ for all $(u=1, \ldots, n-1)$.

The second summand of $L^{c}$ is linear in $\mathbf{Q}$ and therefore also convex in $\mathbf{Q}$. Thus, also the objective function $L=L^{c}+L^{r}$ of the general cost variance optimization problem is convex as a nonnegative linear combination of convex functions if $c_{i n v, u}>c_{i n v, u+1}$ for all $(u=1, \ldots, n-1)$.

\section{B.5. Proof of uniqueness and existence of the portfolio optimum with two technologies}

Proof. Rewriting the optimality condition as given by Eqn. (36) leads to

$$
A\left(\sigma_{1}^{2}+\sigma_{2}^{2}-2 \sigma_{12}\right) Q_{2}-A\left(\sigma_{1}^{2}-\sigma_{12}\right) Q_{E}=\frac{c_{i n v, 1}-c_{i n v, 2}}{t_{1}}+\bar{c}_{o p, 1}-\bar{c}_{o p, 2}
$$


Here, the risk-free term is separated from the risk-term, each to one side of the optimality condition. For brevity, we denote the left hand-side of the latter equation with $l\left(t_{1}\right)$ and the right hand-side with $r\left(t_{1}\right)$, i.e.

$$
\begin{aligned}
l\left(t_{1}\right) & :=A\left(\sigma_{1}^{2}+\sigma_{2}^{2}-2 \sigma_{12}\right) Q_{2}-A\left(\sigma_{1}^{2}-\sigma_{12}\right) Q_{E}, \\
r\left(t_{1}\right) & :=\frac{c_{i n v, 1}-c_{i n v, 2}}{t_{1}}+\bar{c}_{o p, 1}-\bar{c}_{o p, 2} .
\end{aligned}
$$

Because it holds $\left(\sigma_{1}^{2}+\sigma_{2}^{2}-2 \sigma_{12}\right) \geq 0$ for all $\sigma_{1}, \sigma_{2}, \sigma_{12} \geq 0,|\rho| \leq 1$ and since $Q_{2}=\int_{0}^{t_{1}} D(t)-D\left(t_{1}\right) \mathrm{d} t$ is monotone increasing in $t_{1}$, it can be concluded that also $l\left(t_{1}\right)$ is monotone increasing in $t_{1}$, i.e. $\frac{\partial l\left(t_{1}\right)}{\partial t_{1}} \geq 0$. In contrast, it can be seen that $r\left(t_{1}\right)$ is hyperbolically decreasing in $t_{1}$ thus $\frac{\partial r\left(t_{1}\right)}{\partial t_{1}} \leq 0$, given $\left(c_{o p, 1}<\right.$ $\left.c_{o p, 2}\right) \wedge\left(c_{i n v, 1}>c_{i n v, 2}\right)$.

The optimal operating time $t_{1}^{*}$ satisfying condition (36) is given by the intersection of $l\left(t_{1}\right)$ and $r\left(t_{1}\right)$ (see Figure 9). This value represents the optimal operating time of the peak-load technology and captures the trade-off of the variance-minimal and the cost-minimal run-time of the peak load technology. A unique intersection point is obtained if $l(T) \geq r(T)$, i.e.

$$
A Q_{E}\left(\sigma_{2}^{2}-\sigma_{12}\right) \geq \frac{1}{T}\left(c_{i n v, 1}-c_{i n v, 2}\right)+\bar{c}_{o p, 1}-\bar{c}_{o p, 2}
$$

and the two functions will cross exactly once in the interval $[0, T]$, resulting in a unique solution from the optimality condition (36). The latter assumption will generally be fulfilled as empirically shown in Section 4. In the rare case of $A Q_{E}\left(\sigma_{2}^{2}-\sigma_{12}\right)<\frac{1}{T}\left(c_{i n v, 1}-c_{i n v, 2}\right)+\bar{c}_{o p, 1}-\bar{c}_{o p, 2}, l\left(t_{1}\right)$, and $r\left(t_{1}\right)$ have no intersection in the interval $[0, T]$. Hence, in this case there is no interior solution to problem (8) to (11).

\section{B.6. Proof of sensitivity properties of the cost-variance efficient portfolio}

Proof of Property 3.6.2. To derive sensitivity properties of the optimal portfolio fuel mix on the risk attitude factor $A$, we use in the two-technology case the first order derivative of the optimality condition itself. Total differentiation of Eqn. (36) with respect to $A$ and following reallocation leads to

$$
\begin{aligned}
\frac{\mathrm{d} t_{1}^{*}}{\mathrm{~d} A}\left(\bar{c}_{o p, 1}-\bar{c}_{o p, 2}+A Q_{E}\left(\sigma_{1}^{2}-\sigma_{12}\right)-A\left(\sigma_{1}^{2}+\sigma_{2}^{2}-2 \sigma_{12}\right) Q_{2}\right)+ \\
\quad+t_{1}^{*}\left(\left(\sigma_{1}^{2}-\sigma_{12}\right) Q_{E}-Q_{2}\left(\sigma_{1}^{2}+\sigma_{2}^{2}-2 \sigma_{12}\right)+A\left(\sigma_{1}^{2}+\sigma_{2}^{2}-2 \sigma_{12}\right) t_{2} D^{\prime}\left(t_{1}^{*}\right) \frac{\mathrm{d} t_{1}^{*}}{\mathrm{~d} A}\right)=0 \\
\Leftrightarrow \quad \frac{\mathrm{d} t_{1}^{*}}{\mathrm{~d} A}=\frac{\left(t_{1}^{*}\right)^{2}\left(Q_{2}\left(\sigma_{1}^{2}+\sigma_{2}^{2}-2 \sigma_{12}\right)-Q_{E}\left(\sigma_{1}^{2}-\sigma_{12}\right)\right)}{c_{i n v, 2}-c_{i n v, 1}+\left(t_{1}^{*}\right)^{3} A\left(\sigma_{1}^{2}+\sigma_{2}^{2}-2 \sigma_{12}\right) D^{\prime}\left(t_{1}^{*}\right)} .
\end{aligned}
$$

At first, suppose $\frac{\mathrm{d} t_{1}^{*}}{\mathrm{~d} A} \leq 0$. Taking into account the negativity of the denominator in Eqn. (41), this requires consequently the enumerator in the latter term to be non-negative, i.e. $\frac{Q_{2}}{Q_{E}} \geq \frac{\sigma_{1}^{2}-\sigma_{12}}{\sigma_{1}^{2}+\sigma_{2}^{2}-2 \sigma_{12}}$. By inserting 0 as the lower bound of $\frac{Q_{2}}{Q_{E}}$, it follows $\forall \sigma_{1}, \sigma_{2}, \rho$ with $\left(\frac{\sigma_{1}}{\sigma_{2}} \leq \rho\right) \Rightarrow\left(\frac{\mathrm{d} t_{1}^{*}}{\mathrm{~d} A} \leq 0\right)$.

From the upper bound $\frac{Q_{2}}{Q_{E}} \leq 1$ it can be concluded in this case $\left(\frac{\mathrm{d} t_{1}^{*}}{\mathrm{~d} A} \leq 0\right) \Rightarrow\left(\frac{\sigma_{2}}{\sigma_{1}} \geq \rho\right)$. Remark that condition $\frac{\sigma_{1}}{\sigma_{2}} \leq \rho$ can be considered as sufficient and $\frac{\sigma_{2}}{\sigma_{1}} \geq \rho$ as necessary for the case $\frac{\mathrm{d} t_{1}^{*}}{\mathrm{~d} A} \leq 0$. Per definition 


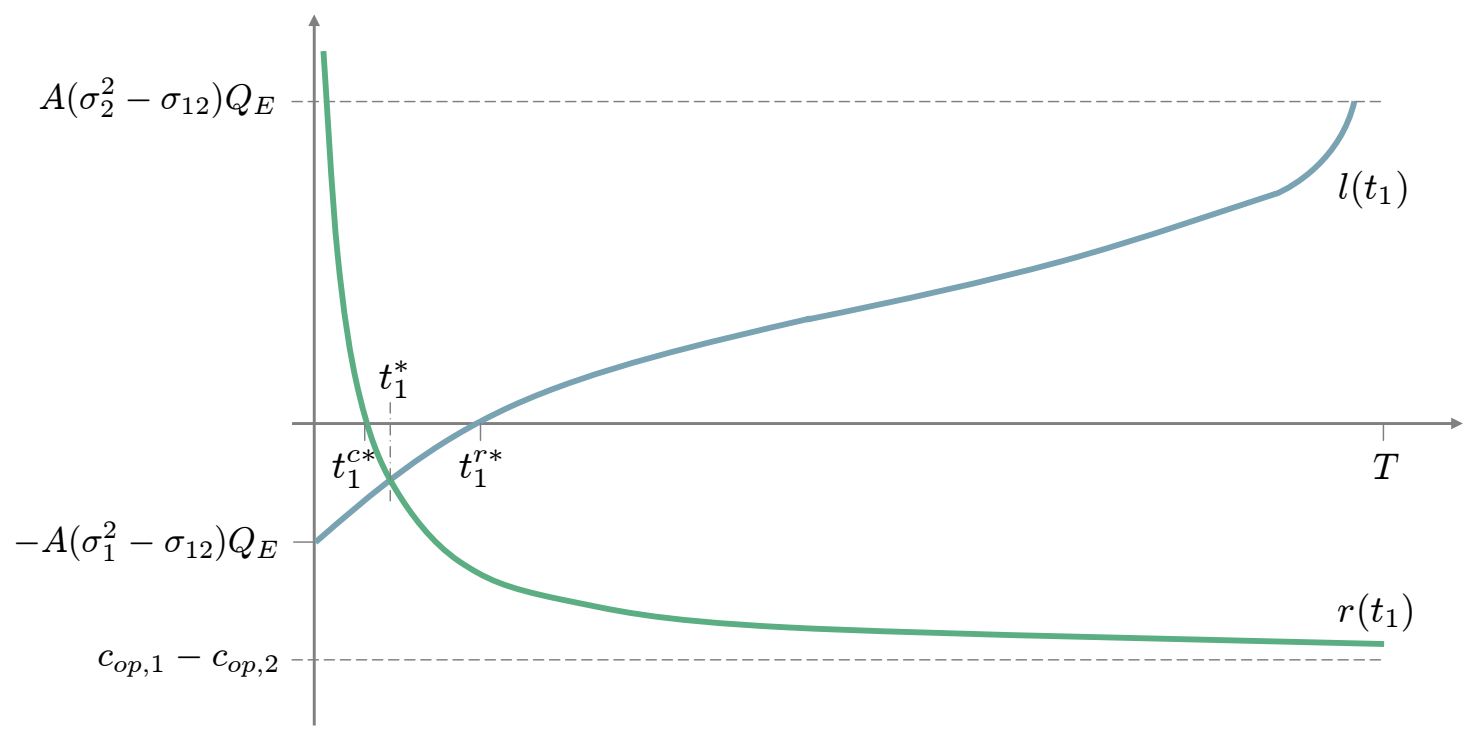

Figure 9: Graphical proof of the uniqueness of results from the optimality condition. The intersection of $r\left(t_{1}\right)$ and $l\left(t_{1}\right)$ represents the optimal operating time.

of the coefficient of correlation it is $|\rho| \leq 1$. Therefore, $\frac{\sigma_{1}}{\sigma_{2}} \leq \rho$ implies $\sigma_{2} \geq \sigma_{1}$. The latter again implies $\frac{\sigma_{2}}{\sigma_{1}} \geq \rho$. Hence, with $\left(\frac{\sigma_{1}}{\sigma_{2}} \leq \rho\right) \Rightarrow\left(\frac{\sigma_{2}}{\sigma_{1}} \geq \rho\right)$, the necessary condition implies the sufficient condition and we can simply state

$$
\left(\frac{\mathrm{d} t_{1}^{*}}{\mathrm{~d} A} \leq 0\right) \Leftrightarrow\left(\frac{\sigma_{1}}{\sigma_{2}} \leq \rho\right) .
$$

Now, the case $\frac{\mathrm{d} t_{1}^{*}}{\mathrm{~d} A} \geq 0$ follows directly from the negation of this equivalence:

$$
\left(\frac{\mathrm{d} t_{1}^{*}}{\mathrm{~d} A} \geq 0\right) \Leftrightarrow\left(\frac{\sigma_{1}}{\sigma_{2}} \geq \rho\right)
$$

Proof of Property 3.6.3. Alternatively, the sensitivity of $t_{1}^{*}$ on the parameter $A$ can be checked by comparison of the optimal operating times of the pure cost-efficient portfolio, $t_{2}^{r *}$, and the pure risk-efficient portfolio, $t_{2}^{r *}:$

$$
\left(\frac{\mathrm{d} t_{1}^{*}}{\mathrm{~d} A} \lesseqgtr 0\right) \Leftrightarrow\left(t_{1}^{c *} \lesseqgtr t_{2}^{r *}\right)
$$

This can be seen from the risk term and the cost term of optimality condition (cf. Eqn. (36)) as previously defined in Eqs. (39) and (40). With $l\left(t_{1}\right)$ monotone increasing and $r\left(t_{1}\right)$ monotone decreasing in $t_{1}$, we can indirectly derive sensitivity properties of $t_{1}^{*}$ from the sensitivities of $l(0), l(T)$, and the variance minimal operating time $t_{1}^{r *}$ with $l\left(t_{1}^{r *}\right)=0$ (cf. Figure 9 ). Knowing that $Q_{2}\left(t_{1}^{r *}\right)$ is monotone increasing in $t_{1}^{r *}$, it can be seen from that the variance minimal operating time is independent from the parameter $A$ :

$$
Q_{2}\left(t_{1}^{r *}\right)=Q_{E} \frac{\sigma_{1}^{2}+\sigma_{2}^{2}-2 \sigma_{12}}{\sigma_{1}^{2}-\sigma_{12}}>0 \quad \text { for } \sigma_{1} \gtrless \sigma_{2} .
$$


Therefore, it holds

$$
\left(\frac{\partial l(0)}{\partial A} \gtreqless 0\right) \wedge\left(t_{1}^{c *}<t_{1}^{r *}\right) \Rightarrow\left(\frac{\partial t_{1}^{*}}{\partial A} \gtreqless 0\right), \quad\left(\frac{\partial l(T)}{\partial A} \lesseqgtr 0\right) \wedge\left(t_{1}^{c *}>t_{1}^{r *}\right) \Rightarrow\left(\frac{\partial t_{1}^{*}}{\partial A} \gtreqless 0\right) .
$$

For $l$ and its partial differentials we can state

$$
\begin{aligned}
& l(0)=-A\left(\sigma_{1}^{2}-\sigma_{12}\right) Q_{E} \lesseqgtr 0 \quad \text { for } \rho \lesseqgtr \frac{\sigma_{1}}{\sigma_{2}}, \quad \frac{\partial l(0)}{\partial A}=-\left(\sigma_{1}^{2}-\sigma_{12}\right) Q_{E} \lesseqgtr 0 \quad \text { for } \rho \lesseqgtr \frac{\sigma_{1}}{\sigma_{2}}, \\
& l(T)=A\left(\sigma_{2}^{2}-\sigma_{12}\right) Q_{E} \gtreqless 0 \quad \text { for } \rho \lesseqgtr \frac{\sigma_{2}}{\sigma_{1}}, \quad \frac{\partial l(T)}{\partial A}=\left(\sigma_{2}^{2}-\sigma_{12}\right) \gtreqless 0 \quad \text { for } \rho \lesseqgtr \frac{\sigma_{2}}{\sigma_{1}} .
\end{aligned}
$$

Note that from $l(0)<0$ follows $\frac{\partial l(0)}{\partial A}<0$, similarly $l(T)>0$ implies $\frac{\partial l(T)}{\partial A}>0$. Thus, within the boundaries where both technologies are part of the pure risk-efficient portfolio, i.e. $\rho \leq \frac{\sigma_{2}}{\sigma_{1}}$ and $\rho \leq \frac{\sigma_{1}}{\sigma_{2}}$ (cf. Property 3.6.1), we can conclude

$$
\left(t_{1}^{c *} \lesseqgtr t_{1}^{r *}\right) \Leftrightarrow\left(\frac{\partial t_{1}^{*}}{\partial A} \gtreqless 0\right)
$$

Proof 1 for Property 3.6.4. Total differentiation of optimality condition Eqn. (36) with respect to $\sigma_{1}$ yields

$$
\begin{aligned}
\frac{\mathrm{d} t_{1}^{*}}{\mathrm{~d} \sigma_{1}} & \left(c_{o p, 1}-c_{o p, 2}+A Q_{E}\left(\sigma_{1}^{2}-\sigma_{12}\right)-A\left(\sigma_{1}^{2}+\sigma_{2}^{2}-2 \sigma_{12}\right) Q_{2}\right)+ \\
& +t_{1}^{*} A\left(\left(2 \sigma_{1}-\sigma_{2} \rho\right) Q_{E}-2 Q_{2}\left(\sigma_{1}-\sigma_{2} \rho\right)+\left(\sigma_{1}^{2}+\sigma_{2}^{2}-2 \sigma_{12}\right) t_{1}^{*} D^{\prime}\left(t_{1}^{*}\right) \frac{\mathrm{d} t_{1}^{*}}{\mathrm{~d} \sigma_{2}}\right)=0 \\
\Leftrightarrow & \frac{\mathrm{d} t_{1}^{*}}{\mathrm{~d} \sigma_{1}}=\frac{\left(t_{1}^{*}\right)^{2} A\left(\left(\sigma_{2} \rho-2 \sigma_{1}\right) Q_{E}-2 Q_{2}\left(\sigma_{2} \rho-\sigma_{1}\right)\right)}{c_{i n v, 2}-c_{i n v, 1}+\left(t_{1}^{*}\right)^{3} A\left(\sigma_{1}^{2}+\sigma_{2}^{2}-2 \sigma_{12}\right) D^{\prime}\left(t_{1}^{*}\right)} .
\end{aligned}
$$

Consider $\frac{\mathrm{d} t_{1}^{*}}{\mathrm{~d} \sigma_{1}} \geq 0$ which requires the enumerator in Eqn. (42) to be negative. Consequently, two cases have to be differentiated:

I) For non-negativity of the term $Q_{2}(\cdot)$ in Eqn. (42), let be $\rho>\frac{\sigma_{1}}{\sigma_{2}}$ : Consequently, $\frac{Q_{2}}{Q_{E}} \geq \frac{\sigma_{2} \rho-2 \sigma_{1}}{2 \sigma_{2} \rho-2 \sigma_{1}}$ has to hold. Using $\frac{Q_{2}}{Q_{E}} \geq 0$ as the lower bound, it follows $\rho \leq \frac{2 \sigma_{1}}{\sigma_{2}}$.

II) For negativity of the term $Q_{2}(\cdot)$ in Eqn. (42), let be $\rho<\frac{\sigma_{1}}{\sigma_{2}}$ : Then $\frac{\mathrm{d} t_{1}^{*}}{\mathrm{~d} \sigma_{1}} \geq 0$ requires $\frac{Q_{2}}{Q_{E}} \leq \frac{\sigma_{2} \rho-2 \sigma_{1}}{2 \sigma_{2} \rho-2 \sigma_{1}}$. Using $\frac{Q_{2}}{Q_{E}} \leq 1$ as the upper bound, it follows $\rho \geq 0$.

Hence, within the boundaries $\frac{\sigma_{1}}{\sigma_{2}}>\rho$ and $\frac{\sigma_{2}}{\sigma_{1}}>\rho$, it can be concluded $\frac{\mathrm{d} t_{1}^{*}}{\mathrm{~d} \sigma_{1}} \geq 0$ for all $\sigma_{1}, \sigma_{2}, \rho \geq 0 .^{29}$

\footnotetext{
${ }^{29}$ For $\frac{\mathrm{d} t t_{1}^{*}}{\mathrm{~d} \sigma_{1}} \leq 0$, we can proceed vice versa to obtain a sufficient condition for $\sigma_{1}, \sigma_{2}, \rho$ fulfilling the assumption. Since we do only obtain the null set, the existence of a parameter set with $\frac{\mathrm{d} t_{1}^{*}}{\mathrm{~d} \sigma_{1}} \leq 0$ remains unproven.
} 
Proof 2 for Property 3.6.4. Total differentiation of Eqn. (36) with respect to $\sigma_{2}$ yields

$$
\begin{aligned}
\frac{\mathrm{d} t_{1}^{*}}{\mathrm{~d} \sigma_{2}} & \left(c_{o p, 1}-c_{o p, 2}+A Q_{E}\left(\sigma_{1}^{2}-\sigma_{12}\right)-A\left(\sigma_{1}^{2}+\sigma_{2}^{2}-2 \sigma_{12}\right) Q_{2}\right)- \\
& -t_{2} A\left(\sigma_{1} \rho Q_{E}+2 Q_{2}\left(\sigma_{2}-\sigma_{1} \rho\right)-\left(\sigma_{1}^{2}+\sigma_{2}^{2}-2 \sigma_{12}\right) t_{1}^{*} D^{\prime}\left(t_{1}^{*}\right) \frac{\mathrm{d} t_{1}^{*}}{\mathrm{~d} \sigma_{2}}\right)=0 \\
\Leftrightarrow \quad \frac{\mathrm{d} t_{1}^{*}}{\mathrm{~d} \sigma_{2}} & =\frac{\left(t_{1}^{*}\right)^{2} A\left(\sigma_{1} \rho Q_{E}+2 Q_{2}\left(\sigma_{2}-\sigma_{1} \rho\right)\right)}{c_{i n v, 2}-c_{i n v, 1}+\left(t_{1}^{*}\right)^{3} A\left(\sigma_{1}^{2}+\sigma_{2}^{2}-2 \sigma_{12}\right) D^{\prime}\left(t_{1}^{*}\right)} .
\end{aligned}
$$

The case $\frac{\mathrm{d} t_{1}^{*}}{\mathrm{~d} \sigma_{2}} \leq 0$ requires the enumerator in Eqn. (43) to be non-negative. It needs to be distinguished between the following cases:

I) For non-negativity of the term $Q_{2}(\cdot)$ in Eqn. (43), let be $\rho<\frac{\sigma_{2}}{\sigma_{1}}$, i.e. $\frac{Q_{2}}{Q_{E}} \geq \frac{\sigma_{1} \rho-2}{2\left(\sigma_{1} \rho-\sigma_{2}\right)}$. Using $\frac{Q_{2}}{Q_{E}} \geq 0$ as the lower bound, it follows $\rho \geq 0$.

II) For negativity of the term $Q_{2}(\cdot)$ in Eqn. (43), let be $\rho>\frac{\sigma_{2}}{\sigma_{1}}$ : Then $\frac{\mathrm{d} t_{1}^{*}}{\mathrm{~d} \sigma_{2}} \leq 0$ requires $\frac{Q_{2}}{Q_{E}} \leq \frac{\sigma_{2} \rho-2 \sigma_{1}}{2 \sigma_{2} \rho-2 \sigma_{1}}$. Using $\frac{Q_{2}}{Q_{E}} \leq 1$ as the upper bound, it follows $\rho \leq \frac{2 \sigma_{2}}{\sigma_{1}}$.

Taken both cases together and considering the boundaries $\frac{\sigma_{1}}{\sigma_{2}}>\rho$ and $\frac{\sigma_{2}}{\sigma_{1}}>\rho$, we obtain for all $\sigma_{1}, \sigma_{2}, \rho \geq 0$, $\frac{\mathrm{d} t_{1}^{*}}{\mathrm{~d} \sigma_{2}} \leq 0 .^{30}$

Proof 3 for Property 3.6.4. As shown, total differentiation of the optimality condition Eqn. (36) with respect to $\rho$ leads to

$$
\begin{aligned}
\frac{\mathrm{d} t_{1}^{*}}{\mathrm{~d} \rho}\left(c_{o p, 1}-c_{o p, 2}+A Q_{E}\left(\sigma_{1}^{2}-\sigma_{12}\right)-A\left(\sigma_{1}^{2}+\sigma_{2}^{2}-2 \sigma_{12}\right) Q_{2}\right)- \\
-t_{1}^{*} A\left(\sigma_{1} \sigma_{2} Q_{E}-2 \sigma_{1} \sigma_{2} Q_{2}-\left(\sigma_{1}^{2}+\sigma_{2}^{2}-2 \sigma_{12}\right) t_{2} D^{\prime}\left(t_{2}\right) \frac{\mathrm{d} t_{1}^{*}}{\mathrm{~d} \rho}\right)=0 \\
\Leftrightarrow \frac{\mathrm{d} t_{1}^{*}}{\mathrm{~d} \rho}=\frac{\left(t_{1}^{*}\right)^{2} A \sigma_{1} \sigma_{2}\left(Q_{1}-Q_{2}\right)}{c_{i n v, 2}-c_{i n v, 1}+\left(t_{1}^{*}\right)^{3} A\left(\sigma_{1}^{2}+\sigma_{2}^{2}-2 \sigma_{12}\right) D^{\prime}\left(t_{1}^{*}\right)}
\end{aligned}
$$

For $\frac{\mathrm{d} t_{1}^{*}}{\mathrm{~d} \rho} \leq 0$, it can be reasoned from Eqn. (44) that $\left(Q_{1}-Q_{2} \geq 0\right) \Leftrightarrow\left(\frac{Q_{2}}{Q_{E}} \leq \frac{1}{2}\right)$ has to be fulfilled. As seen in proof 2 , if $\frac{\sigma_{1}}{\sigma_{2}} \geq \rho$ the relation $\frac{Q_{2}}{Q_{E}} \leq \frac{\sigma_{1}^{2}-2 \sigma_{12}}{\sigma_{1}^{2}+\sigma_{2}^{2}-\sigma_{12}}$ holds and represents an upper bound for the quotient $\frac{Q_{2}}{Q_{E}}$. Thus, we can conclude

$$
\left(\frac{\sigma_{1}^{2}-\sigma_{1} \sigma_{2} \rho}{\sigma_{1}^{2}+\sigma_{2}^{2}-2 \sigma_{1} \sigma_{2} \rho} \leq \frac{1}{2}\right) \Leftrightarrow\left(\sigma_{1} \leq \sigma_{2}\right)
$$

Hence, within the boundaries $\frac{\sigma_{1}}{\sigma_{2}}>\rho$ and $\frac{\sigma_{2}}{\sigma_{1}}>\rho$, we can conclude $\frac{\mathrm{d} t_{1}^{*}}{\mathrm{~d} \sigma_{1}} \leq 0$ for all $\sigma_{1}, \sigma_{2} \geq 0$ with $\sigma_{1} \leq \sigma_{2}$.

\footnotetext{
${ }^{30}$ Applying the analogue estimation for $\frac{\mathrm{d} t_{1}^{*}}{\mathrm{~d} \sigma_{2}} \geq 0$, however, cannot prove the existence of a feasible set of $\sigma_{1}, \sigma_{2}, \rho$ as we only obtain the null set.
} 


\section{References}

Awerbuch, S., 2004. Towards a finance-oriented valuation of conventional and renewable energy sources in Ireland. Report, Sustainable Energy Ireland.

URL http://www.awerbuch.com/shimonpages/shimondocs/Ireland_SEI-Jun-25-04.doc

Awerbuch, S., 2006. Portfolio-based electricity generation planning: Policy implications for renewables and energy security. Mitigation and adaptation strategies for global change 11 (3), 693-710.

Awerbuch, S., Berger, M., 2003. Applying portfolio theory to EU electricity planning and policy-making. Report number EET/2003/03, IEA.

URL https://www.iea.org/textbase/papers/2003/port.pdf

Awerbuch, S., Stirling, A., Jansen, J., Beurskens, L., 2006. Full-spectrum portfolio and diversity analysis of energy technologies. In: Leggio, K., Bodde, D., Taylor, M. (Eds.), Managing Enterprise Risk. Elsevier Global Energy Policy and Economics Series. Elsevier Science Ltd, pp. 202-222.

BAFA, 2009. Bafa energy statistics. Bundesamt für Wirtschaft und Ausfuhrkontrolle (German Federal Office of Economics and Export Control).

URL http://www.bafa.de/

Balagopal, B., Gilliland, G., 2005. Integrating value and risk in portfolio strategy for energy companies. The Boston Consulting Group.

URL http://www.bcg.com/impact_expertise/publications/files/Integrating_Value_Risk_Energy_Companies_Sep2005. pdf

Bar-Lev, D., Katz, S., 1976. A portfolio approach to fossil fuel procurement in the electric utility industry. The Journal of Finance $31(3), 933-47$.

BMWi, 2008. Gross power generation by energy sources. Bundesministerium für Wirtschaft und Technologie (German Federal Ministry of Economics and Technology).

URL http://www. bmwi.de/

Boiteux, M., 1960. Peak load pricing. Journal of Business 33, 157-179.

Crew, M., Kleindorfer, P., 1986. The economics of public utility regulation. Macmillan.

Delarue, E., De Jonghe, C., Belmans, R., D'haeseleer, W., 2009. Applying portfolio theory on the electricity sector: Installed capacity versus actual electricity generation. 10th IAEE European Conference, Vienna, Austria, September 8-10th, 2009.

Deutscher Bundestag, 2002. Gesetz zur geordneten Beendigung der Nutzung der Atomenergie zur gewerblichen Erzeugung von Elektrizität. Bundesgesetzblatt Jahrgang 2002, Teil I Nr. 26, published on April 26th, 2006, 1351-1359.

URL http://www.bgblportal.de/BGBL/bgbl1f/bgbl102026s1351.pdf

ECX, 2009. ECX historical data. European Climate Exchange.

URL http://www.ecx.eu/

Elishakoff, I., 1999. Probabilistic Theory of Structures. Dover Publications.

Fischer, R., Serra, P., 2003. Energy prices in the presence of plant indivisibilities. Energy Economics 25 (4), $303-314$.

Gotham, D., Muthuraman, K., Preckel, P., Rardin, R., Ruangpattana, S., 2009. A load factor based mean-variance analysis for fuel diversification. Energy Economics 31, 249-256.

Horn, R., Johnson, C., 1985. Matrix Analysis.

Humphreys, H., McClain, K., 1998. Reducing the impacts of energy price volatility through dynamic portfolio selection. Energy Journal 19 (3), 107-131.

IEA, 2008. World energy outlook. International Energy Agency, OECD.

Jansen, J., Beurskens, L., Tilburg, X., 2006. Application of portfolio analysis to the Dutch generating mix. Reference case and 
two renewables cases. Report c-05-100, ECN.

URL http://www.ecn.nl/publications/PdfFetch.aspx?nr=ECN-C--05-100

Kleindorfer, P., Li, L., 2005. Multi-period var-constrained portfolio optimization with applications to the electric power sector. Energy Journal 26 (1), 1-26.

Konstantin, P., 2009. Praxisbuch Energiewirtschaft: Energieumwandlung, -transport und -beschaffung im liberalisierten Markt. Springer.

Krey, B., Zweifel, P., 2006. Efficient, diversified and secure electricity generation portfolios for Switzerland and the United States. Working Paper No. 0602, Scioeconomic Institute, University of Zurich, also in: Florence IAEE European Conference, 2007

URL http://www.soi.uzh.ch/research/wp/2006/wp0602.pdf

Lange, O., 1942. The foundations of welfare economics. Econometrica 10, 215-228.

Lemming, J., Meibom, P., 2003. Including investment risk in large-scale power-market models. Energy \& Environment 14 (5), $599-626$.

Markowitz, H., 1952. Portfolio selection. The Journal of Finance 7 (1), 77-91.

Markowitz, H., 1959. Portfolio Selection: Efficient Diversification of Investments, Cowles Foundation Monography 16. Yale University Press, New Haven, CT.

Oren, S., 2000. Capacity payments and supply adequacy in competitive electricity markets. In: Proceedings of the VII Symposium of Specialists in Electric Operational and Expansion Planning. pp. 21-26.

Pigou, A., 1932. Economics of welfare. Macmillan, London.

Roques, F., Newbery, D., Nuttall, W., 2008. Fuel mix diversification incentives in liberalized electricity markets: A meanvariance portfolio theory approach. Energy Economics 30 (4), 1831-1849.

Roques, F., Nuttall, W., Newbery, D., 2006a. Using probabilistic analysis to value power generation investments under uncertainty. EPRG Working Paper.

URL www.electricitypolicy.org.uk

Roques, F., Nuttall, W., Newbery, D., de Neufville, R., 3 2006b. Nuclear power: a hedge against uncertain gas and carbon prices? Cambridge Working Papers in Economics 0555.

Sachverständigenrat, 2008. Energy prices in Germany. Sachverständigenrat zur Begutachtung der gesamtwirtschaftlichen Entwicklung (German Council of Economic Experts).

URL http://www.sachverstaendigenrat-wirtschaft.de

Schneeweiss, H., 1965. Konsequenzen des Bernoulli-Prinzips für die Präferenzstruktur von Normalverteilungen. Mathematical Methods of Operations Research 9 (4), 238-249.

StaBu, 2009. Data on energy price trends - long-time series from January 2000 to December 2008. Statistisches Bundesamt German Federal Statistical Office.

URL http://www.ec-destatis.de/

Stirling, A., 1994. Diversity and ignorance in electricity supply investment - addressing the solution rather than the problem. Energy Policy 22 (3), 195-216.

Tobin, J., 1958. Liquidity preference as behavior towards risk. Review of Economic Studies 25 (2), 65-86.

UCTE, 2009. Consumption data, hourly load values Germany. Union for Co-ordination of Transportation of Electricity.

URL http://www.ucte.org/resources/dataportal/consumption/

UxC, 2009. Historical ux month-end spot prices. The Ux Consulting Company, LLC.

URL http://www.uxc.com/

Weber, C., 2005. Uncertainty in the Electric Power Industry: Methods and Models for Decision Support. Springer.

Weber, C., Swider, D., 2004. Power plant investments under fuel and carbon price uncertainty. 6th IAEE European Conference 
on Modelling in Energy Economics and Policy.

White, B., Lesser, J., Lowengrub, P., Yang, S., 2007. A mean-variance portfolio optimization of California's generation mix to 2020. Draft Report for California Energy Commission.

URL http://www.bateswhite.com/news/pdf/2007_July_CEC_Report.pdf 